\title{
A feedback loop between heterochromatin and the nucleopore complex controls germ- cell to oocyte transition during Drosophila oogenesis
}

\author{
Kahini Sarkar ${ }^{1}$, Noor M Kotb ${ }^{1,2}$, Alex Lemus ${ }^{1}$, Elliot T Martin ${ }^{1}$, Alicia McCarthy ${ }^{1,3}$, Justin \\ Camacho $^{1}$, Ayman lqbal ${ }^{1}$, Alex M. Valm ${ }^{1}$, Morgan A Sammons ${ }^{1}$, Prashanth Rangan ${ }^{1 *}$ \\ 1Department of Biological Sciences/RNA Institute, University at Albany SUNY, Albany, NY \\ 12222 \\ 2Department of Biomedical Sciences, The School of Public Health, University at Albany SUNY, \\ Albany, NY 12222 \\ ${ }^{3}$ Current address: 10x Genomics Headquarters, 6230 Stoneridge Mall Rd, Pleasanton, CA \\ 94588 \\ *Corresponding Author and Lead Contact: prangan@albany.edu
}

\section{Summary}

Germ cells differentiate into oocytes that become totipotent upon fertilization. How the highly specialized oocyte acquires this distinct cell fate is poorly understood. During Drosophila oogenesis, H3K9me3 histone methyltransferase SETDB1 translocates from the cytoplasm to the nucleus of germ cells concurrent with oocyte specification. Here, we discovered that nuclear SETDB1 is required to silence a cohort of differentiation-promoting genes by mediating their heterochromatinization. Intriguingly, SETDB1 is also required for the upregulation of 18 of the 30 nucleoporins (Nups) that comprise the nucleopore complex (NPC). NPCs in turn anchor SETDB1dependent heterochromatin at the nuclear periphery to maintain $\mathrm{H} 3 \mathrm{~K} 9 \mathrm{me} 3$ and gene silencing in the egg chambers. Aberrant gene expression due to loss of SETDB1 or Nups results in loss of oocyte identity, cell death and sterility. Thus, a feedback loop between heterochromatin and NPCs promotes transcriptional reprogramming at the onset of oocyte specification that is critical to establish oocyte identity.

\section{Introduction}

Germ cells give rise to gametes that upon fertilization launch the next generation (Cinalli et al., 2008; Seydoux and Braun, 2006; Spradling et al., 2011). In the gonad, germ cells become germline stem cells (GSCs) that self-renew and differentiate to give rise to sperm or an oocyte (Gilboa and Lehmann, 2004; Kershner et al., 2013; Ko et al., 2010; Lesch and Page, 2012; Reik and Surani, 2015; Seydoux and Braun, 2006). The oocyte, upon fertilization or by parthenogenesis, can differentiate into every cell lineage in the adult organism and thus has a capacity to be totipotent (Ben-Ami and Heller, 2005; Lehmann, 2012; Riparbelli et al., 2017; Yuan and Yamashita, 2010). The gene regulatory mechanisms that enable the transition from germ cells to oocytes are not fully understood. 
Drosophila has a well-characterized transition from germline stem cell (GSC) to an oocyte (Dansereau and Lasko, 2008; Gilboa and Lehmann, 2004; Spradling et al., 2011; Allan C Spradling, 1993). Drosophila ovaries comprise individual units called ovarioles that house the GSCs in a structure called the germarium (Figure 1A-A1) (Lehmann, 2012; Xie and Spradling, 2000). GSC division results in a new GSC (self-renewal) and a cystoblast, which differentiates via incomplete mitotic divisions, giving rise to 2-, 4-, 8- and 16-cell cysts (Figure 1A1) (Chen and McKearin, 2003a, 2003b; Xie, 2013). One of these 16 cells is specified as the oocyte whereas the other 15 cells become nurse cells (Huynh and St Johnston, 2004; Koch et al., 1967; Navarro et al., 2004). Somatic cells envelop the nurse cells and the specified oocyte to form an egg chamber (Figure 1A1) (Xie and Spradling, 2000). The nurse cells produce mRNAs, called maternal mRNAs, that are deposited into the specified oocyte mediated by an RNA binding protein, Egalitarian (Egl) (Blatt et al., 2020; Kugler and Lasko, 2009; Lilly and Spradling, 1996; Mach and Lehmann, 1997; Navarro et al., 2004; A C Spradling, 1993). An inability to specify or maintain the oocyte fate leads to death of the egg chamber mid-oogenesis and, in turn, sterility (Blatt et al., 2021; Navarro et al., 2004).

The transition from GSC to an oocyte requires dynamic changes in gene expression that promote progressive differentiation (Flora et al., 2017). Once a GSC gives rise to the cystoblast, it expresses differentiation factor Bag of marbles (Bam), promoting its differentiation to an 8-cell cyst (McKearin and Ohlstein, 1995; McKearin and Spradling, 1990). In the 8-cell cyst, the expression of the RNA binding fox-1 homolog 1 (Rbfox 1 ) is required to mediate transition into the 16-cell cyst stage, allowing for an oocyte to be specified (Carreira-Rosario et al., 2016). Translation of Rbfox1 requires increased levels of ribosomal small subunit protein 19 (RpS19) accomplished in part by expression of the germline specific paralog $R p S 19 b$ in the undifferentiated and early differentiating stages (McCarthy et al., 2019). During differentiation, the germline also initiates meiotic recombination mediated by the synaptonemal complex consisting of proteins such as Sisters Unbound (Sunn), Corona (Cona) and Orientation Disruptor (Ord) (Ables, 2015; Cahoon and Hawley, 2016; Hughes et al., 2018; Orr-Weaver, 1995; Page and Hawley, 2001). More than one cell in the cyst stage initiates recombination but as oocyte differentiation proceeds, only the specified oocyte retains the synaptonemal complex (Figure 1A1) (Ables, 2015; Orr-Weaver, 1995; Page and Hawley, 2001). After oocyte-specification, the levels of mRNAs encoding RpS19b and some synaptonemal complex proteins are diminished, suggesting early oogenesis genes are no longer expressed (McCarthy et al., 2019). How the expression of these early oogenesis genes is attenuated is not known.

In Drosophila, the SET Domain Bifurcated Histone Lysine Methyltransferase 1 (SETDB1) (also called Eggless) is required for deposition of gene silencing Histone H3 Lysine 9 trimethylation (H3K9me3) marks and heterochromatin formation (Clough et al., 2014, 2007; Rangan et al., 2011; Yoon et al., 2008). SETDB1 is expressed throughout Drosophila oogenesis, but as the oocyte is specified, it shifts from a cytoplasmic to predominantly nuclear localization (Clough et al., 2007). A conserved cofactor called Windei (Wde) is required for either nuclear translocation, nuclear stability, or targeting of SETDB1 to its target loci (Koch et al., 2009; Osumi et al., 2019).

88 Loss of SETDB1 during germline development results in an accumulation of undifferentiated cells 
(Rangan et al., 2011; Smolko et al., 2018). In addition, loss of SETDB1 and wde also result in egg chambers that do not grow in size and die mid-oogenesis (Clough et al., 2014; Koch et al., 2009). SETDB1 is known to be required for silencing transposons and male-specific transcripts in the female germline (Czech et al., 2018; Rangan et al., 2011; Smolko et al., 2018). However, neither the upregulation of transposons nor male-specific genes in female germline result in egg chambers that do not grow in size (Malone et al., 2009; Shapiro-Kulnane et al., 2015; Smolko et al., 2020). Together these data suggests that SETDB1 silences a yet-unidentified group of genes to promote oogenesis.

97

Here, we find that genes that are expressed in early stages of oogenesis, including genes that promote oocyte differentiation and synaptonemal complex formation, are silenced upon oocyte specification, via a feedback loop between SETDB1-mediated heterochromatin and the

101 nucleopore complex (NPC). Inability to silence these differentiation-promoting genes due to loss of either SETDB1 or members of the NPC results in loss of oocyte identity and death. Several aspects of germ cell differentiation have been studied and have been implicated in loss of fertility

104 in sexually reproducing organisms. Our work indicates that a previously unappreciated broad transcriptional reprogramming silences critical aspects of the germ cell differentiation program at the onset of oocyte specification and is essential to promote oocyte identity.

\section{Results}

\section{SETDB1 promotes silencing of $R p S 19 b$ reporter at the onset of oocyte specification}

We hypothesized that the expression of early oogenesis mRNAs such as RpS19b is silenced upon oocyte specification. To monitor RpS19b expression, we used a reporter that expresses an RpS19b-GFP fusion from the endogenous RpS19b promoter. This RpS19b-GFP shows high expression in the germarium and attenuated expression post-oocyte specification and in the subsequent egg chambers, consistent with its endogenous RpS19b mRNA expression pattern (Figure 1B-C1, G) (Jevitt et al., 2020; McCarthy et al., 2019).

Using a previously characterized hemagglutinin (HA) tagged endogenous SETDB1, we found that a large fraction of SETDB1 translocates from the cytoplasm to the nucleus concurrent with oocyte specification (Figure S1A-A3) (Seum et al., 2007). To test if SETDB1 is required for the silencing of RpS19b (Clough et al., 2014, 2007), we performed germline knockdown (GKD) of SETDB1, in the background of RpS19b-GFP reporter. We detected the germline, RpS19b-GFP, and spectrosomes/fusomes/somatic cell membrane in ovaries by immunostaining for Vasa, GFP, and 1B1, respectively (Lasko and Ashburner, 1988; Zaccai and Lipshitz, 1996). We found that, compared to the control, GKD of SETDB1 resulted in ectopic RpS19b-GFP protein expression in the differentiated egg chambers without affecting levels in the undifferentiated stages (Figure 1CG; Figure S1B). Thus, SETDB1 is required for repression of RpS19b-GFP reporter in the differentiated egg chambers. 
133 the differentiated stages of oogenesis without affecting cytoplasmic levels in the undifferentiated 134 stages (Figure S1D-F). Whereas GKD of SETDB1 reduced H3K9me3 throughout oogenesis, 135 GKD of wde reduced $\mathrm{H} 3 \mathrm{~K} 9 \mathrm{me} 3$ only in the differentiated egg chambers but not in the 136 undifferentiated stages (Figure S1G-J). We found that GKD of wde, like GKD of SETDB1, results 137 in ectopic RpS19b-GFP protein expression in the egg chambers without affecting levels in the 138 undifferentiated stages (Figure 1C-G). In addition to upregulation of RpS19b-GFP, GKD of both SETDB1 and wde resulted in egg chambers that did not grow in size and died mid-oogenesis as previously reported (Figure S1K) (Clough et al., 2014; Koch et al., 2009). Thus, repression of the RpS19b-GFP reporter in the differentiated egg chambers requires nuclear SETDB1.

SETDB1 and Wde repress genes that are primarily expressed prior to oocyte specification To determine if SETDB1 and Wde repress other differentiation-promoting genes in addition to RpS19b, we performed RNA Sequencing (RNA-seq). We compared ovaries from SETDB1- and wde- GKD flies to ovaries from wild-type (WT) flies, including young virgin flies which lack latestage egg chambers. Principal component analysis of the RNA-seq data revealed that SETDB1 and wde ovary transcriptomes closely resembles young virgin WT rather than adult WT (Figure S2A). Using a 1.5-fold cut off (Fold Change (FC) $\geq|1.5|$ ) and False Discovery Rate (FDR)<0.05, we found that compared to young virgin WT control, 2316 genes were upregulated and 1972 were downregulated in SETDB1 GKD ovaries, and 1075 genes were upregulated and 442 were downregulated in wde-GKD ovaries (Figure 2A-B) (Supplemental Table 1). Moreover, comparing wde- to SETDB1- GKD ovaries showed significant overlap of the upregulated $(80 \%)$ and downregulated (75\%) transcripts, suggesting that SETDB1 and Wde co-regulate a cohort of genes during oogenesis (Figure 2C; Figure S2B).

SETDB1 and Wde are known to repress gene expression, thus we first focused on mRNAs with increased levels in the GKD ovaries (Clough et al., 2014; Osumi et al., 2019). Gene Ontology (GO) analysis of the shared upregulated RNAs indicated that many were genes involved in differentiation (Figure 2D). Among the upregulated RNAs was $R p S 19 b$, validating our initial screen, as well as genes that promote synaptonemal complex formation such as sunn, ord and cona (Figure 2E; Figure S2C-E). In addition, the blanks mRNA, which is highly expressed only in GSCs, cystoblasts and early cysts of WT, was upregulated and ectopically expressed in the egg chambers of SETDB1- and wde-GKD ovaries (Figure 2F; Figure S2F-I) (Blatt et al., 2021). Blanks is a component of a nuclear siRNA pathway that has critical roles in the testis but does not have any overt function during oogenesis (Gerbasi et al., 2011). Thus, SETDB1 and wde repress a cohort of RNAs that are either critical for transition from GSC to an oocyte or merely expressed during early oogenesis.

To determine when during oogenesis SETDB1 and Wde act to repress genes, we analyzed available RNA-seq libraries that were enriched for GSCs, cystoblasts, and cysts, early egg chambers and late-stage egg chambers (McCarthy et al., 2019). We found that SETDB1/wderegulated RNAs decreased after the cyst stages and their levels were attenuated in the later stages of oogenesis compared to non-targets (Figure 2G, Figure S2J-L) (McCarthy et al., 2019). This reduction did not happen in absence of SETDB1 and wde (Figure 2G). RNA in situ analysis of blanks, and RpS19b revealed that these mRNAs are present in the early stages of oogenesis 
and are attenuated after oocyte specification in controls but that these RNAs persisted in SETDB1 and wde GKD egg chambers (Figure 2H-O). Thus, mRNAs that are broadly expressed prior to oocyte specification, become repressed by SETDB1 and Wde in differentiated egg chambers.

\section{SETDB1 represses transcription of a subset of targets by increasing $\mathrm{H} 3 \mathrm{~K} 9 \mathrm{me} 3$ enrichment} To investigate whether SETDB1/Wde-regulated mRNAs are repressed at the level of transcription, we examined a subset of nascent mRNAs (pre-mRNAs) by qRT-PCR. Indeed, the levels of nascent RpS19b, ord, sunn, cona and blanks mRNAs were increased in SETDB1/wdeGKDs ovaries compared to control WT ovaries (Figure S3A-B). These data suggest that transcription of these genes increases upon loss of SETDB1 or Wde.

To determine if the SETDB1-dependent repression of these genes involves changes in H3K9me3, we performed CUT\&RUN (Ahmad, 2018; Skene and Henikoff, 2017) on adult WT ovaries enriched for differentiated egg chambers where these genes are repressed (Figure 2G). Analysis of CUT\& RUN data from adult WT showed enrichment of H3K9me3 marks on previously identified SETDB1 targets and genes containing heterochromatin such as PHD Finger Protein 7 (phf7) and light (It) respectively validating our CUT\&RUN data (Figure 3A-B; Figure S3C) (Devlin et al., 1990; Smolko et al., 2018). As genes in Drosophila genome are closely packed, we only analyzed the gene body from 5'UTR to the end of the 3'UTR to unambiguously identify SETDB1 regulated genes (Schwartz and Cavalli, 2017). We found that 1593 out of 2,316 genes upregulated upon loss of SETDB1 are enriched for H3K9me3 marks compared to IgG negative control (Figure 3C). In addition, we found that 888 genes lose H3K9me3 on their gene bodies upon GKD of SETDB1 including RpS19b and ATP-dependent chromatin assembly factor (Acf) (Figure 3D-F). The upregulated genes that do not show changes to H3K9me3 marks within the gene body may be regulated by elements outside of the gene body or indirectly. Importantly, taken together, our data suggest that SETDB1 is required for H3K9me3 enrichment and transcriptional repression of a cohort of early-oogenesis genes in the egg chamber.

SETDB1 is required for transposon repression during oogenesis (Andersen et al., 2017; Rangan et al., 2011), and the upregulation of transposons can affect gene expression (Sienski et al., 2012; Upadhyay et al., 2016). However, we found that the upregulation of genes in the differentiated stages that we observed upon depletion of SETDB1 was not due to the secondary effect of transposon upregulation as the expression of $R p S 19 b$ reporter was not altered in germline depleted of aubergine (aub), a critical component of the piRNA pathway (Figure S3D-F) (Chen et al., 2007; Czech et al., 2018; Malone et al., 2009; Wang et al., 2015). Nor, did aub depletion cause mid-oogenesis death as we observed in SETDB1 and wde GKDs (Figure S3D-F) (Chen et al., 2007; Wilson et al., 1996). Overall, our data suggest that loss of SETDB1 derepresses a subset of genes during late oogenesis via decreased H3K9me3, independent of transposon dysregulation.

\section{SETDB1 is required for the expression of NPC components}

GO term analysis of downregulated targets of SETDB1/wde GKD included genes that regulate transposition, consistent with the previously described role of SETDB1/Wde in the piRNA pathway and those that regulate proper oocyte development, consistent with the previously described 
phenotype (Figure 4A)(Andersen et al., 2017; Clough et al., 2007; Koch et al., 2009; Rangan et al., 2011).

Unexpectedly, we observed that genes involved in nucleocytoplasmic transport were downregulated in SETDB1/wde-GKD ovaries as compared to controls (Figure 4A). Nucleocytoplasmic transport is mediated by Nucleopore complexes (NPCs), which span the nuclear membrane and consist of a cytoplasmic ring, a central scaffold spanning the nuclear envelope, and a nuclear ring and basket (Figure 4B) (M. Capelson et al., 2010; Doucet and Hetzer, 2010; Gozalo and Capelson, 2016). Beyond regulating nucleocytoplasmic transport, NPCs also regulate gene transcription, for instance by anchoring and maintaining heterochromatic domains (Capelson and Hetzer, 2009; Hou and Corces, 2010; Iglesias et al., 2020; Sarma and Willis, 2012; Sood and Brickner, 2014). We found that GKD of SETDB1/wde in the germline resulted in downregulation of 18 out of $\sim 30$ nucleoporins (Nups) that make up the Nucleopore complex in Drosophila (Figure 4C), including a germline enriched Nup154 that is critical for oogenesis (Colozza et al., 2011; Gigliotti et al., 1998; Grimaldi et al., 2007). The Nups that were downregulated upon depletion of SETDB1 and wde were not isolated to one specific NPC subcomplex (Figure 4B-C).

We found that nascent mRNAs corresponding to the SETDB1/Wde targets Nup154, Nup205 and Nup107 were downregulated in SETDB1/wde-GKD ovaries, whereas the non-target Nup62 was unaffected, suggesting that SETDB1/Wde promotes transcription of a cohort of Nups (Figure 4D). In addition, the levels of a Nup107-RFP fusion protein, under endogenous control (Katsani et al., 2008), were significantly reduced in the cysts and egg chambers of SETDB1- and wde-GKD compared to controls (Figure S4A-D).

To determine if loss of Nup expression in SETDB1/wde-GKD ovaries resulted in loss of NPC formation, we performed immunofluorescence with an antibody that is known to mark NPCs in Drosophila (Maya Capelson et al., 2010; Davis and Blobel, 1987; Hampoelz et al., 2019; Kuhn et al., 2019). We found that NPC levels were reduced in the egg chambers of SETDB1/wde-GKD ovaries compared to controls (Figure 4E-H), but the nuclear lamina was unaffected (Figure S4E$\mathrm{H}$ ), and NPCs in the soma were also unaffected (Figure 4I). Thus, SETDB1/wde are required for the proper expression of Nups and NPC formation after oocyte specification.

Heterochromatic genes and piRNA clusters require heterochromatin to promote their transcription (Rangan et al., 2011; Weiler and Wakimoto, 1995). Although we found that SETDB1 is required for upregulation of Nups, CUT\&RUN analysis of H3K9me3 marks revealed that only 3 of the Nup genes had any enrichment of H3K9me3 (Mbo, Nup188, Gp210). Moreover, among SETDB1regulated Nups, only Gp210 showed any heterochromatic enrichment (Supplemental Table 2). Taken together, we find that SETDB1 promotes proper expression of Nups by a yet unknown mechanism in the germline.

264

Nucleoporins are required to maintain heterochromatin domains at the nuclear periphery Our data so far indicate that, in Drosophila female germline, heterochromatin formation mediated by SETDB1 is required for proper NPC formation by promoting proper expression of a subset of 
Nups including Nup107 and Nup154 (Figure 4C). In yeast, a subset of Nups are part of the heterochromatin proteome and are required to cluster and maintain heterochromatin at the NPC (Iglesias et al., 2020). This subset includes Nup107 and the yeast homolog of Nup154, Nup155, which both have reduced expression in SETDB1/Wde-GKD compared to controls. We hypothesized that in Drosophila, SETDB1 could promote silencing of early oogenesis genes by promoting heterochromatin formation. This heterochromatin then promotes expression of Nups and NPC formation, which can then help maintain heterochromatin by anchoring it to nuclear periphery and thus promoting silencing of early-oogenesis genes.

To first determine if heterochromatin and nucleoporins associate in Drosophila female germline, we utilized antibody against H3K9me3 to mark heterochromatin and Nup107-RFP to mark NPCs in WT ovarioles (Katsani et al., 2008; Rangan et al., 2011). We found that H3K9me3 domains were often at the nuclear periphery, in close proximity with Nup107-RFP (Figure 5A-A2, E). Next, to determine if loss of Nups leads to loss of heterochromatin, we first depleted Nup154 and probed for heterochromatin formation. We chose Nup154, as its loss of function phenotype of Nup154 has been well described (Gigliotti et al., 1998; Grimaldi et al., 2007). We found that GKD of Nup154 in the germline, resulted in egg chambers that do not grow and die mid-oogenesis as previously described for Nup154 mutants (Figure S5A-B2) (Gigliotti et al., 1998). In addition, depletion of Nup154 results in proper translocation of SETDB1 from the cytoplasm to the nucleus suggesting that transport of SETDB1 into the nucleus is not grossly affected (Figure S5C-D1). By staining for H3K9me3 marks, we found that upon GKD of Nup154, heterochromatin domains initially form (Figure S5E-F2). However, in the egg chambers of Nup 154 GKD, the colocalization between H3K9me3 domains and Nup107-RFP levels at the nuclear periphery were significantly reduced prior to significant reduction of heterochromatin levels (Figure 5A-D1, Figure S5E-F3, I). GKD of Nup107 also resulted in egg chambers that do not grow and loss of heterochromatin (Figure S5E-I). Thus, Nups 154 and 107, which are positively regulated by SETDB1, are required for H3K9me3 localization at the nuclear periphery for H3K9me3 maintenance in the female germline.

\section{Nups are required for silencing early-oogenesis genes}

Based on our findings above that Nups are required to maintain H3K9me3 levels and localization, we hypothesized that they are also required to silence the early-oogenesis RNAs in differentiated egg chambers. To test this hypothesis, we depleted Nup154 and Nup107 in the germline of a fly carrying the RpS19b-GFP reporter. We found that GKD of these nucleoporins resulted in upregulation of RpS19b-GFP phenocopying GKD of SETDB1/wde (Figure 6A-C; Figure S6AB1, D). Moreover, germline depletion of Nup62, which is within the NPC but not regulated by SETDB1, also resulted in upregulation of RpS19bGFP and egg chambers that did not grow (Figure S6A-D). This suggests that activity of NPC components and not just the Nups regulated by SETDB1 are required for silencing RpS19b-GFP reporter.

308

To determine if Nups are required for silencing other early oogenesis RNAs, we performed RNAseq, and compared Nup154 GKD ovaries with young ovaries as a developmental control (Figure S2A). Using a 1.5 -fold cut off (Fold Change $(F C) \geq|1.5|$ ) and False discovery rate $(F D R)<0.05$ ), we found that compared to control, in Nup154 GKD 2809 genes are upregulated, and 2922 genes 
are downregulated (Figure 6D) (Supplemental Table 1). Strikingly, 97\% of upregulated genes and $89 \%$ of downregulated SETDB1/Wde targets overlapped with Nup154 GKD (Figure 6E; S6E). Nup154 was involved in silencing genes that promote oocyte differentiation including synaptonemal complex components ord, sunn and cona as well as RpS19b (Figure 6F; S6F-H). In addition, GKD of Nup154 also resulted in upregulation of blanks (Figure 6G). The levels of Nup154-regulated RNAs decreased after the cyst stage, when the oocyte is specified, in contrast to non-targets, which have similar RNA levels at all stages (Figure 6H; S6I-J). Thus, Nup154 is critical for silencing early-oogenic mRNAs in the differentiated egg chambers.

To determine if Nup154 is required for H3K9me3 marks at SETDB1-regulated gene, loci such as RpS19b, we carried out CUT\& RUN for H3K9me3 in control and Nup154 GKD. We found that 564 out of 622 genes displaying a loss in H3K9me3 in Nup154 GKD also show the same loss in SETDB1 GKD including RpS19b and Acf (Figure 6I; S6K-L) (Supplemental Table 2). Taken together, we find that Nups are required for silencing and H3K9me3 at a subset of SETDB1/Wderegulated loci.

\section{Silencing genes expressed during the early oogenesis stages is required for maintaining oocyte fate}

We next asked why loss of SETDB1, wde and Nups results in egg chambers that do not grow and die mid-oogenesis. Egg chambers with oocyte specification or maintenance defects result in death of egg chambers mid-oogenesis (Blatt et al., 2021). To determine if there are oocyte specification or maintenance defects, we stained GKD of SETDB1, wde and Nup154 for the oocyte marker Egalitarian (Egl) as well as Vasa and 1B1 (Mach and Lehmann, 1997; Navarro et al., 2004). In the early stages of oogenesis, as in control, GKD of SETDB1, wde and Nup154 resulted in one Egl positive cell, suggesting that oocyte is specified (Figure 7A-E). While initial Egl localization to oocytes appeared to be normal, we cannot rule out subtle specification defects. However, in the later egg chambers, compared to control ovariole, GKD of SETDB1, wde and Nup154 resulted in either mis-localization or diffuse Egl expression suggesting loss of oocyte fate (Figure 7A-E). Taken together, these data suggest that SETDB1, Wde and Nup154 are required for maintaining the oocyte fate.

\section{Discussion}

Many maternally contributed mRNAs in oocytes are critical for early development after fertilization (Calvi et al., 1998; Huynh and St Johnston, 2004; Kugler and Lasko, 2009; Navarro et al., 2004; Telfer, 1975). We previously showed that many mRNAs expressed in germ cells and the undifferentiated stages of oogenesis must be selectively degraded and thus excluded from the maternal contribution (Blatt et al., 2021). However, the potential role of transcriptional silencing of germ cell and GSC-enriched genes during oogenesis was unclear. Here, we found that regulated translocation of SETDB1 into the nucleus during oocyte specification is required to silence germ cell- and early oogenesis-genes in the differentiated egg chambers (Figure 7F), and that this process is essential to maintain oocyte fate. Thus, some genes that are expressed in germ cells and some that promote differentiation are transcriptionally silenced at the onset of oocyte specification mediated by a feedback loop between heterochromatin and NPC. 
Regulated heterochromatin formation during oocyte specification promotes germ cell to oocyte transition

A large fraction of SETDB1 is cytoplasmic in the undifferentiated stages of the germline. As the oocyte is being specified during differentiation, SETDB1 becomes mostly nuclear (Clough et al., 2014). This translocation of SETDB1 to the nucleus during oocyte specification is mediated by Windei (Wde), the Drosophila ortholog of mAM/MCAF1 (Koch et al., 2009; Osumi et al., 2019). Here we find that translocation of SETDB1 to the nucleus during oocyte specification is required to silence germ cell and early-oogenesis genes at the onset of oocyte specification. MCAF1 also regulates the accumulation of SETDB1 in the nucleus in mammalian cells (Tsusaka et al., 2019). In addition, loss of SETDB1 during mammalian oogenesis results in meiotic defects and infertility (Eymery et al., 2016). These data suggest that regulated heterochromatin formation is conserved to promote proper oogenesis in mammals.

We discovered that SETDB1 is required to silence two major classes of genes. The first group is involved in GSC differentiation into an oocyte, including critical genes that promote meiosis I. The second group of genes are those that are merely expressed in the germ cells prior to differentiation into an oocyte, but have no specific function in the female germline such as blanks (Blatt et al., 2021; Gerbasi et al., 2011). We propose that these genes that are silenced upon oocyte specification are either detrimental to late oogenesis or early embryogenesis. Indeed, it has been shown that overexpression of one such gene actin $57 B$ (act57B), which is repressed by SETDB1/Wde (Supplemental Table 1), is detrimental to oogenesis (Blatt et al., 2021; Duan et al., 2020). Remarkably, some of the mRNAs encoded by genes that are transcriptionally silenced by SETDB1 during this transition are also targeted at the post-transcriptional level for degradation by members of the no go decay pathway such as blanks and Act57B (Blatt et al., 2021). Thus, our data suggests that the regulation of gene expression during the germ cell to oocyte transition reflects a two-step process: transcriptional silencing dependent on SETDB1, and posttranscriptional degradation of mRNAs to exclude a cohort of germ cell mRNAs from the maternal contribution (Blatt et al., 2021).

SETDB1 is guided to its target transposons and piRNA clusters mediated by piRNAs (Andersen et al., 2017; Czech et al., 2018; Koch et al., 2009; Osumi et al., 2019). However, our data suggests that the piRNA pathway does not play a part in silencing germ cell and early oogenesis RNAs. We find that loss of aub does not result in upregulation of RpS19b. This result is consistent with the fact that loss of aub and piwi in the germline does not result in egg chambers that do not grow (Chen et al., 2007; Wilson et al., 1996). In somatic cells of the gonad, loss of wde function eliminates nuclear SETDB1 signal (Osumi et al., 2019; Timms et al., 2016). However, upon depletion of Wde, SETDB1 was still ubiquitinated, a requirement for its nuclear retention (Osumi et al., 2019). This suggests that in absence of Wde, SETDB1 can translocate to the nucleus but cannot find its targets. Osumi et al. (2019) suggested that Wde could mediate SETDB1 recruitment to the its targets, leading to $\mathrm{H} 3 \mathrm{~K} 9$ me 3 deposition. In mammals, it has been shown that transcriptional factors such as the KRAB domain-containing Zinc finger proteins recruit SETDB1 to the target genes for silencing, but such transcription factors have not been identified in the female gonad (Frietze et al., 2010; Schultz et al., 2002). Thus, SETDB1 targets germ cell 
and early oogenesis genes for silencing independent of the piRNA pathway but through a yet undetermined mechanism, either through Wde or through yet undetermined transcription factors.

Nucleopore complex and heterochromatin are in a feedback loop to promote gene silencing

402 The NPC not only mediates selective nucleo-cytoplasmic transport of macromolecules but also regulates gene expression by anchoring chromatin domains, including heterochromatin to the nuclear periphery (Maya Capelson et al., 2010; M. Capelson et al., 2010; Holla et al., 2020; Iglesias et al., 2020; Sarma and Willis, 2012). In addition, in yeast, several Nups are also part of the heterochromatin proteome suggesting that NPCs can regulate gene expression by regulating heterochromatin (Brickner et al., 2019; Iglesias et al., 2020). Consistent with these observations, we find that in the female germline of Drosophila, NPC and heterochromatin are closely associated. Loss of NPCs due to depletion of individual Nups results in loss of heterochromatin and subsequent upregulation of germ cell and early oogenesis genes resulting in oogenesis defects. The $97 \%$ overlap of target genes between SETDB1, wde and Nup154 is indicative that Nups are functioning in the same pathway as SETDB1. This suggests that in the female germline, not only do NPCs associate with heterochromatin, but that NPCs also play a role in maintaining heterochromatin and gene repression during germ cell to oocyte transition.

Silencing of early oogenesis genes at the onset of oocyte specification is timed with exit from mitotic cell cycle. Drosophila nucleopore complex consists of $\sim 30$ different nucleoporins some of which are solubilized during early mitotic cell division (Güttinger et al., 2009; Laurell and Kutay, 2011). Nucleoporins are recruited to the chromatin in early anaphase followed by sequential reassembly of the complex (Kiseleva et al., 2001; Kutay et al., 2021). During Drosophila oogenesis, in the premeiotic stage, the GSC divides to eventually produce a 16-cell cyst (Huynh and St Johnston, 2004; Koch et al., 1967; Lehmann, 2012; Spradling et al., 2011). Prophase-I of meiosis begins in cysts where the oocyte is also specified (Ables, 2015; Orr-Weaver, 1995). We find that nucleoporins promote silencing of genes that are required for initiation of meiosis I such as Rbfox 1 and synaptonemal complex components ord, sunn and cona once the cysts have stopped dividing and the oocyte is being specified. Taken together, our data suggests a mechanism wherein after the mitotic division of cysts have ceased and meiosis I is initiated, the reassembly of NPC simultaneously promotes silencing of the genes required for the transition from mitotic GSC division to meiotic oocyte specification.

While NPC association with heterochromatin has been described, remarkably we find that loss of heterochromatin results in attenuated expression of some but not all Nup mRNAs. Heterochromatic genes and piRNA clusters require heterochromatin to promote their transcription (Andersen et al., 2017; Devlin et al., 1990; Rangan et al., 2011). However, by analyzing CUT \&RUN data and previously published ChIP data of H3K9me3 marks, we found that only one Nup regulated by SETDB1 is enriched for H3K9me3 marks. Therefore, SETDB1 indirectly promotes expression of Nups.

The number of genes that need to be silenced varies based on cell types and developmental trajectory. How levels of heterochromatin are coupled to their NPC docking sites in the cell was 
not known. Like heterochromatin levels, the number of NPCs also varies by cell type and during differentiation (McCloskey et al., 2018). How NPC number is regulated during development was not fully understood. Our findings in the female germline suggest an elegant tuning mechanism for heterochromatin and its NPC docking sites. Heterochromatin promotes levels of NPC which then promote heterochromatin maintenance by tethering it to the nuclear periphery. We find that this loop can be developmentally regulated by controlling levels of SETDB1 in the nucleus mediated by conserved protein Wde to promote heterochromatin formation.

\section{Acknowledgements:}

We are grateful to all members of the Rangan laboratory for discussion and comments on the manuscript. We also thank Dr. Thomas Hurd and Dr. Miler Lee for their comments on the manuscript. We would like to thank Sontheimer lab for Blanks antibody, Lehmann lab for Egl antibody, Bloomington Drosophila Stock Center, Vienna Drosophila Resource Center, Transgenic GKD Project (NIH/NIGMS R01-GM084947), The BDGP Gene Disruption Project, and FlyBase for fly stocks and reagents. Furthermore, we would like to thank CFG Facility at the University at Albany (UAlbany) for performing RNA-seq analyses. P.R. is funded by $\mathrm{NIH/NIGMS} \mathrm{(RO1GM11177} \mathrm{and} \mathrm{RO1GM135628).} \mathrm{M.A.S} \mathrm{is} \mathrm{funded} \mathrm{by} \mathrm{NIH} \mathrm{NIGMS} \mathrm{R35} 138120$ and A.V. is funded by R01DE030927.

\section{Materials and Methods}

\section{Fly lines}

The following RNAi stocks were used in this study; if more than one line is listed, then both were quantitated and the first was shown in the main figure: SETDB1 RNAi (Perrimon lab, (Rangan et al., 2011), Wde RNAi (Bloomington \#33339), Nup154 RNAi (Bloomington \#34710), Nup93 RNAi (VDRC \#V16189), Nup62 RNAi (Bloomington \#35695), Nup107 RNAi (Bloomington \#43189), Nup205 RNAi (VDRC \#V38608).

The following tagged lines were used in this study: dSETDB1-HA (Bontron Lab) (Seum et al., 472 \#35516).

473 The following tissue-specific drivers and double balancer lines were used in this study: UAS-

474 Dcr2;nosGAL4 (Bloomington \#25751), nosGAL4;MKRS/TM6 (Bloomington \#4442), and 475 If/CyO;nosGAL4 (Lehmann Lab).

\section{Reagents for fly husbandry}

477 Flies were grown at $25-29^{\circ} \mathrm{C}$ and dissected between $0-3$ days post-eclosion. 
Fly food was made using the procedures as previously described (summer/winter mix) and narrow vials (Fisherbrand Drosophila Vials; Fischer Scientific) were filled to approximately 10-12mL

480 (Flora et al., 2018).

\section{Dissection and Immunostaining}

482 Ovaries were dissected and teased apart with mounting needles in cold PBS and kept on ice. All incubation was done with nutation. Samples were fixed for 10 minutes in 5\% methanol-free formaldehyde. Ovaries were washed in $0.5 \mathrm{~mL}$ PBT (1X PBS, 0.5\% Triton X-100, 0.3\% BSA) 4 times for 5 minutes each. Primary antibodies in PBT were added and incubated at $4{ }^{\circ} \mathrm{C}$ nutating overnight. Samples were next washed 3 times for 5 minutes each in $0.5 \mathrm{~mL}$ PBT, and once in 0.5 $\mathrm{mL} \mathrm{PBT}$ with $2 \%$ donkey serum (Sigma) for 15 minutes. Secondary antibodies were added in PBT with $4 \%$ donkey serum and incubated at room temperature for 3-4 hours. Samples were washed 3 times for 10 minutes each in $0.5 \mathrm{~mL}$ of 1 X PBST $(0.2 \%$ Tween 20 in $1 \times$ PBS) and incubated in Vectashield with DAPI (Vector Laboratories) for 1 hour before mounting.

The following primary antibodies were used: mouse anti-1B1 (1:20; DSHB), Rabbit anti-Vasa (1:1,000; Rangan Lab), Chicken anti-Vasa (1:1,000; Rangan Lab) (Upadhyay et al., 2016), Rabbit anti-GFP (1:2,000; abcam, ab6556), Rabbit anti-H3K9me3 (1:500; Active Motif, AB_2532132), Mouse anti-H3K27me3 (1:500; abcam, ab6002), Rabbit anti-Egl (1:1,000; Lehmann Lab), Mouse anti-NPC (1:2000; BioLegend, AB_2565026) and Rat anti-HA (1:500; Roche, 11867423 001). The following secondary antibodies were used: Alexa 488 (Molecular Probes), Cy3 and Cy5 (Jackson Labs) were used at a dilution of 1:500.

\section{$498 \quad$ Fluorescence Imaging}

499 The tissues were visualized, and images were acquired using a Zeiss LSM-710 confocal 500 microscope under 20X, 40X and 63X oil objective with pinhole set to 1 airy unit. All gain, laser 501 power, and other relevant settings were kept constant for any immunostainings being compared. 502 Image processing was done using Fiji and gain adjustment and cropping was performed in 503 Photoshop CC 2019.

\section{Colocalization analysis}

Confocal images of control and Nup154-RNAi mutants labeled for RFP-Nup107, H3K9me3, and DAPI were imported into Bitplane Imaris 9.6 .2 for 3D reconstruction and colocalization analysis. Colocalization between RFP-Nup107 and H3K9me3 was calculated on a per egg chamber basis using the Surface-surface coloc function of Imaris and an automatic threshold detection and the surface-to-surface coloc function. The number of colocalized voxels was then normalized to the

\section{Egg laying assays}


Assays were conducted in vials with 3 control or experimental females under testing and 1 wild type control males. Crosses were set up in triplicate for both control and experimental. All flies were 1-day old post-eclosion upon setting up the experiment. Cages were maintained at $29^{\circ} \mathrm{C}$ and plates were changed daily for counting. Analyses were performed for 5 consecutive days. Number of eggs laid were counted and averaged. Adult flies eclosed were counted from all the vials and averaged.

\section{RNA isolation}

Ovaries from flies were dissected in cold 1x PBS. RNA was isolated using TRIzol (Invitrogen, 15596026) (Blatt et al., 2021; McCarthy et al., 2019).

RNA was treated with DNase (TURBO DNA-free Kit, Life Technologies, AM1907), and then run on a $1 \%$ agarose gel to check integrity of the RNA.

RNA-seq library preparation and analysis

Libraries were prepared using the Biooscientific kit. To generate mRNA enriched libraries, total RNA was treated with poly(A)tail selection beads (Bioo Scientific Corp., NOVA-512991). Manufacturer's instructions of the NEXTflex Rapid Directional RNA-seq Kit (Bioo Scientific Corp., NOVA-5138-08) were followed, but RNA was fragmented for 13 minutes. Library quality was assessed with a Fragment Analyzer (5200 Fragment Analyzer System, AATI, Ankeny, IA, USA) following manufacturer's instructions. Single-end mRNA sequencing (75 base pair reads) was performed on biological duplicates from each genotype on an Illumina NextSeq500 by the Center for Functional Genomics (CFG).

After quality assessment, the sequenced reads were aligned to the Drosophila melanogaster genome (UCSCdm6) using HISAT2 (version 2.1.0) with the RefSeq-annotated transcripts as a guide (Kim et al., 2015). Differential gene expression was assayed by DeSeq2, using a false discovery rate (FDR) of 0.05 , and genes with 2 -fold or higher were considered significant. The raw and unprocessed data for RNA-seq generated during this study are available at Gene Expression Omnibus (GEO) databank under accession number: XXX. GO term enrichment on differentially expressed genes was performed using Panther (Thomas et al., 2006).

\section{Fluorescent in situ hybridization}

542 A modified in situ hybridization procedure for Drosophila ovaries was followed. Probes were 543 designed and generated by LGC Biosearch Technologies using Stellaris ${ }^{\circledR}$ RNA FISH Probe 544 Designer, with specificity to target base pairs of target mRNAs. Ovaries (3 pairs per sample) were dissected in RNase free $1 X$ PBS and fixed in $1 \mathrm{~mL}$ of $5 \%$ formaldehyde for 10 minutes. The 546 samples were then permeabilized in $1 \mathrm{~mL}$ of Permeabilization Solution (PBST+1\% Triton-X) rotating in RT for 1 hour. Samples were then washed in wash buffer for 5 minutes $(10 \%$ deionized 548 formamide and $10 \%$ 20x SSC in RNase-free water). Ovaries were covered and incubated 549 overnight with $1 \mathrm{ul}$ of probe in hybridization solution (10\% dextran sulfate, $1 \mathrm{mg} / \mathrm{ml}$ yeast tRNA, 2 
mM RNaseOUT, $0.02 \mathrm{mg} / \mathrm{ml} \mathrm{BSA,} \mathrm{5x} \mathrm{SSC,} \mathrm{10 \%} \mathrm{deionized} \mathrm{formamide,} \mathrm{and} \mathrm{RNase-free} \mathrm{water)} \mathrm{at}$ $30^{\circ} \mathrm{C}$. Samples were then washed 2 times in $1 \mathrm{~mL}$ wash buffer for 30 minutes and mounted in

552 Vectashield.

\section{CUT\&RUN assay}

Ovaries from flies were dissected in ice cold 1x PBS and ovarioles were separated by teasing after dissection with mounting needles. PBS was removed and the samples were permeabilized in $1 \mathrm{~mL}$ of Permeabilization Solution (PBST+1\% Triton-X) rotating in RT for 1 hour. Samples were then incubated overnight at $4^{\circ} \mathrm{C}$ in primary antibody dilutions in freshly prepared BBT+ buffer (PBST + 1\% BSA + $0.5 \mathrm{mM}$ Spermidine + 2 mM EDTA + 1 large Roche complete EDTA-free tablets). Primary antibody was replaced with BBT+ buffer and quickly washed twice. Samples were then incubated in $700 \mathrm{ng} / \mathrm{ml}$ of pAG-MNase in BBT+ buffer rotating for 4 hours at $25^{\circ} \mathrm{C}$. Samples were then quickly washed twice in wash+ buffer $(20 \mathrm{mM} \mathrm{HEPES} \mathrm{pH7.5+150} \mathrm{mM} \mathrm{NaCl}$ $+0.1 \%$ BSA + $0.5 \mathrm{mM}$ Spermidine + 1 large Roche complete EDTA-free tablets in water). Samples were resuspended in $150 \mu \mathrm{l} \mathrm{Wash}+\mathrm{C}$ (wash $++100 \mathrm{mM} \mathrm{CaCl}$ ) and incubated for 45 minutes on nutator at $4^{\circ} \mathrm{C}$. The cleavage reaction was terminated by addition of $150 \mu \mathrm{L}$ StopR ( $\mathrm{NaCl}$ final $200 \mathrm{mM}+$ EDTA final $20 \mathrm{mM}+100 \mu \mathrm{g} / \mathrm{mL}$ RNaseA) and incubating the sample at $37^{\circ} \mathrm{C}$ for 30 minutes. Samples were then centrifuged at $16,000 \times \mathrm{g}$ for 5 minutes and $300 \mu \mathrm{l}$ of the supernatant was collected for DNA discovery. To the supernatant, $2 \mu \mathrm{L} 10 \%$ SDS and $2.5 \mu \mathrm{L}$ of $20 \mathrm{mg} / \mathrm{mL}$ Proteinase $\mathrm{K}$ was added and incubated at $50^{\circ} \mathrm{C}$ for 2 hours. Half of this was kept as a backup and half was used in bead cleanup. $20 \mu \mathrm{L}$ AmpureXP bead slurry and $280 \mu \mathrm{L}$ MXP buffer $(20 \%$ PEG $8000+2.5 \mathrm{M} \mathrm{NaCl}+10 \mathrm{mM} \mathrm{MgCl} 2$ in water) was added to the sample and mixed thoroughly followed by 15 minutes incubation at RT. The beads were separated by magnet and supernatant was discarded. The beads were carefully washed with $80 \%$ ethanol for 30 seconds, while on the magnetic stand and air dried for 2 minutes. The beads were then resuspended in 10 $\mu \mathrm{L}$ DNase free water.

DNA seq library preparation and analysis

The samples from CUT\&RUN assay were used for library preparation using NEBNext ${ }^{8}$ Ultra $^{\text {TM }}$ DNA Library Prep Kit for Illumina ${ }^{\circledR}(E 7645$, E7103) and adaptor ligated DNA were prepared without size selection.

\section{CUT\&Run Data Analysis}

580 Cut\&Run libraries were sequenced as paired-end 75bp reads on the Illumina NextSeq 500 at the 581 University at Albany Center for Functional Genomics. FASTQ files were aligned to the dm6 582 reference genome using HISAT2 (10.1038/s41587-019-0201-4) (-X 10 -I 1000 -no-spliced583 alignment, --no-discordant). Mapping statistics and data will be available from Gene Expression 584 Omnibus. Alignment files were sorted and indexed using samtools and were subsequently used to create bigwig files for visualization with deeptools (--binSize 10)( 10.1093/nar/gkw257). 586 Principle component analysis between samples was performed using the multiBigwigSummary 587 and plotPCA modules from deeptools. Only gene bodies were considered and problematic 
genomic regions (blacklist) were removed from the analysis (10.1038/s41598-019-45839-z). Raw

589 read counts of $\mathrm{H} 3 \mathrm{~K} 9 \mathrm{me} 3$ enrichment across gene bodies was calculated using the HOMER 590 annotateRepeats function and differential enrichment was calculated using DESeq2 (HOMER

591 PMID:20513432, DESeq2 citation 10.1186/s13059-014-0550-8). H3K9me3 occupied genes are 592 those with differential enrichment of H3K9me3 compared to IgG matched control conditions using 593 DESeq2.

\section{Quantitative Real Time-PCR (qRT-PCR)}

$5951 \mu \mathrm{L}$ of cDNA from each genotype was amplified using $5 \mu \mathrm{L}$ of SYBR green Master Mix, $0.3 \mu \mathrm{L}$ of $59610 \mu \mathrm{M}$ of each reverse and forward primers in a $10 \mu \mathrm{L}$ reaction. The thermal cycling conditions 597 consisted of $50^{\circ} \mathrm{C}$ for 2 minutes, $95^{\circ} \mathrm{C}$ for 10 minutes, 40 cycles at $95^{\circ} \mathrm{C}$ for 15 seconds, and $60^{\circ} \mathrm{C}$ 598 for 60 seconds. The experiments were carried out in technical triplicate and minimum 2 biological 599 replicates for each sample. To calculate fold change in mRNA levels, comparison was done to $600 \mathrm{rp49}$ mRNA levels which was used as the control gene. Average of the $2^{\wedge} \Delta \mathrm{Ct}$ for the biological 601 replicates was calculated. Error bars were plotted using standard error of the ratios and $P$-value 602 was determined by Students t-test. 

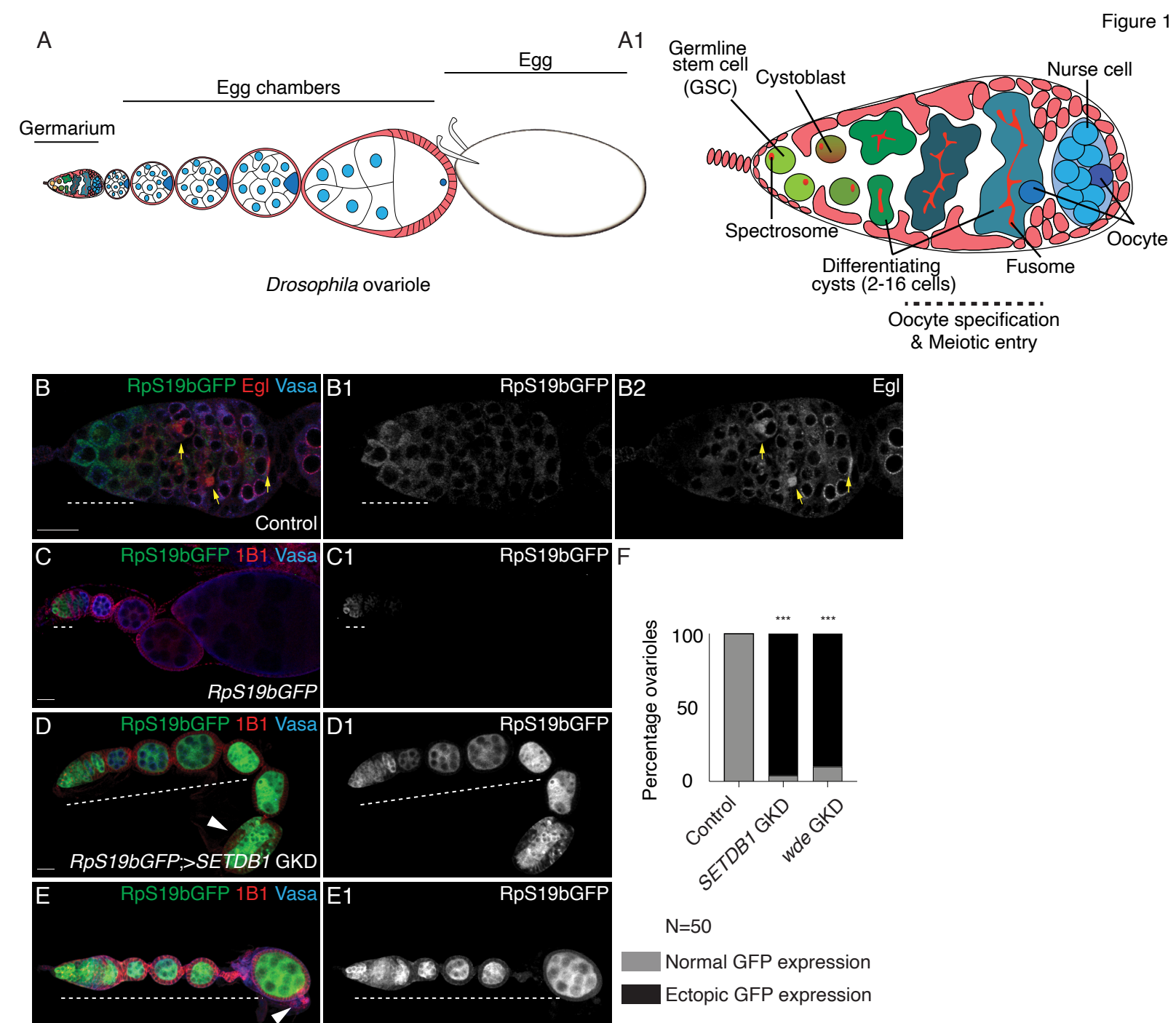

$$
\mathrm{N}=50
$$

$R p S 19 b G F P ;>$ wde GKD

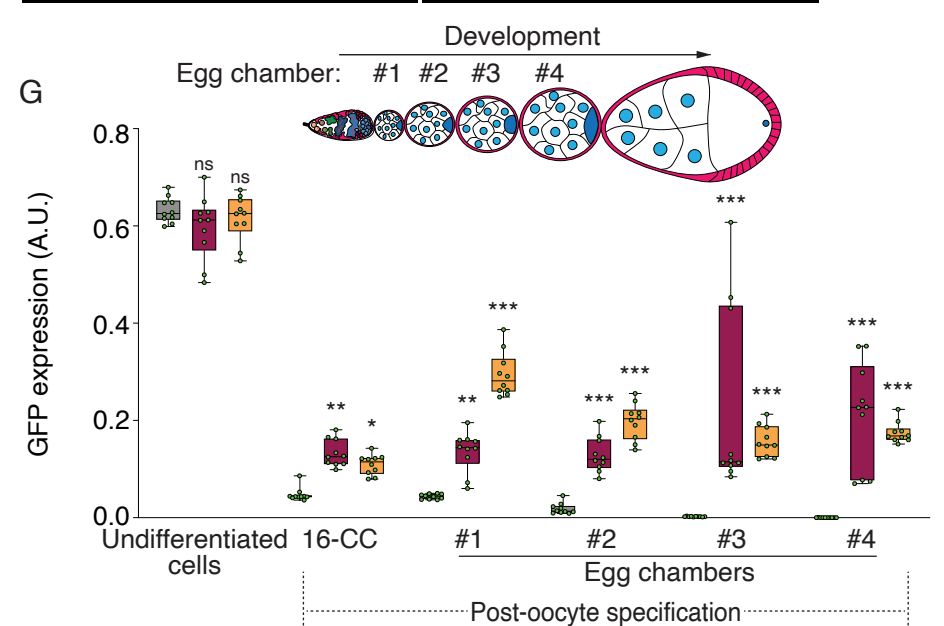

Normal GFP expression Ectopic GFP expression 
(A) A schematic of a Drosophila ovariole. The ovariole consists of germarium and egg chambers corresponding to distinct developmental stages. Egg chambers are connected by somatic cells 610 (white).

(A1) A schematic of a Drosophila germarium, where germline stem cells (GSCs; light green) are at close proximity to somatic niche (red). The GSC divides to give rise to daughter cells called cystoblasts (dark green) which turns on a differentiation program. Both GSCs and cystoblasts are marked by spectrosomes (red). Cystoblasts undergo four incomplete mitotic divisions, giving rise to 2-, 4-, 8-, and 16-cell cysts (green), marked by fusomes (red). During the cyst stages germ cells progress through meiotic cell cycle (prophase I). Upon 16-cell cyst formation, a single cell is specified as the oocyte (dark blue) while the other 15 cells become nurse cells (light blue).

(B-B2) Confocal images of a germarium of a fly carrying $R p S 19 b-G F P$ reporter transgene stained for GFP (green, right grayscale), Egl (red, right grayscale) and Vasa (blue). GFP is expressed in the single cells undifferentiated stages and early cyst stages (white dashed line), while Egl is expressed in the differentiated cysts and localized to the specified oocyte (yellow arrows).

(C-E) Ovariole of control RpS19b-GFP (D-D1), GKD of SETDB1 (E-E1) and wde (F-F1) stained for GFP (green, right grayscale), Vasa (blue) and 1B1 (red). Depletion of these genes resulted in characteristic phenotype of egg chambers not growing and mid oogenesis death (white solid arrows). In addition, ectopic expression of RpS19b-GFP was observed in the egg chambers (white dashed line).

(F) Quantification of ectopic RpS19b-GFP expression upon GKD of SETDB1 or wde compared to control ovaries ( $\mathrm{N}=50$ ovarioles; $96 \%$ in SETDB1 GKD and 90\% in wde GKD compared to 0\% in control.) Statistical analysis was performed with Fisher's exact test on ectopic GFP expression; $* * *=p<0.001$.

(G) Arbitrary units (A.U.) quantification of RpS19b-GFP expression in the germarium and egg chambers during development upon GKD of SETDB1 (magenta) or wde (orange) compared to control ovaries (black). GFP is expressed higher in single cells in the germarium, decreases in the cyst stages, and then attenuated upon egg chamber formation. In SETDB1 and wde GKD, GFP expression persists in the egg chambers. Statistical analysis was performed with Dunnett's multiple comparisons test; $N=10$ ovarioles; $n s=p>0.05,{ }^{*}=p<0.05,{ }^{* *}=p<0.01,{ }^{* * *}=p<0.001$. 

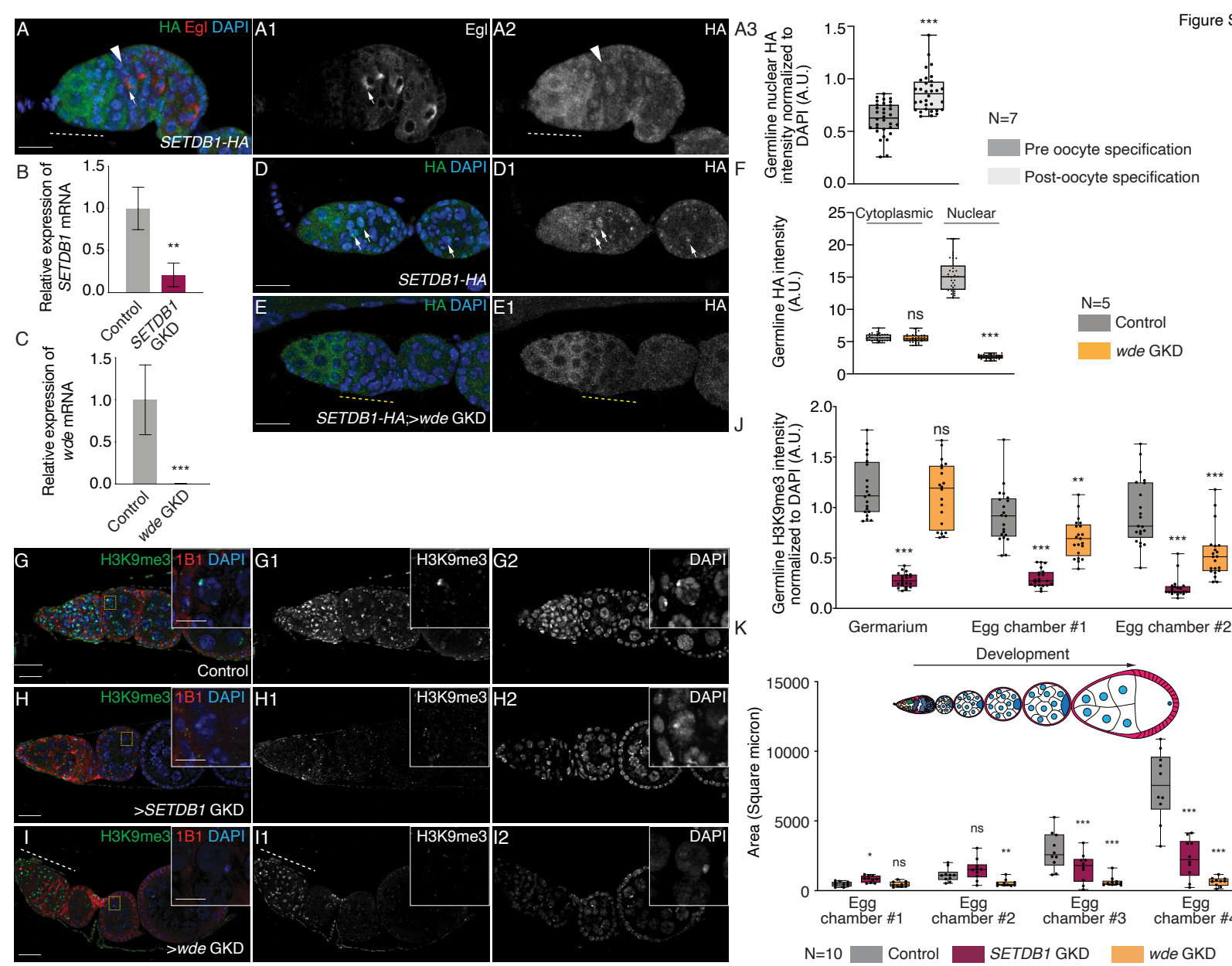

Supplementary Figure1: SETDB1/Wde mediated heterochromatin formation is required for silencing $R p S 19 b$ reporter

(A-A3) Germarium of a fly carrying HA tagged SETDB1 stained for HA (green, right grayscale), oocyte marker Egl (red, right grayscale) and DAPI (blue). White arrows point at the specified oocyte. SETDB1 translocates from the cytoplasm (white dotted line) to the nucleus concurrent with oocyte specification (white solid arrow). Quantitation of HA level (A3) expressed as a ratio of nuclear SETDB1 to DAPI. Statistical analysis was performed with Welch's t-test; $\mathrm{N}=7$ germaria; ns $=p>0.05,{ }^{*}=p<0.05,{ }^{* *}=p<0.01,{ }^{* * *}=p<0.001$ and wde GKD respectively, normalized to control rp49 mRNA levels and indicating knockdown of these genes $\left(\mathrm{N}=3,{ }^{* *}=\mathrm{p}<0.01,{ }^{* *}=\mathrm{p}<0.001\right.$, Error bars are SEM, Student's t-test $)$. HA (green, right grayscale) and Vasa (blue). Yellow arrows point at nuclear HA. GKD of wde shows that levels of HA in the nucleus is attenuated (yellow dotted line). (F) Quantification of germline HA levels in the cytoplasm in the undifferentiated stages and in the nucleus of the 
670 differentiated stages in the germarium in ovaries depleted of wde (orange) compared to control 671 ovaries (gray). Statistical analysis was performed with Welch's t-test; $N=5$ ovarioles; $n s=p>0.05$, $672 \quad{ }^{*}=p<0.05,{ }^{* *}=p<0.01,{ }^{* * *}=p<0.001$.

673

674 (G-J) Ovariole of control UAS-Dcr2;nosGAL4 (G-G1), GKD of SETDB1 (H-H1) or wde (I-I1) 675 stained for H3K9me3 (green, right grayscale), DAPI (blue, right grayscale) and 1B1 (red). Nurse 676 cells from egg chamber highlighted by a dashed yellow square represent cells shown in the inset. 677 Control shows H3K9me3 is present throughout oogenesis in the germline. GKD of SETDB1 678 shows loss of $\mathrm{H} 3 \mathrm{~K} 9 \mathrm{me} 3$ in all stages of the germline while depletion of wde results in decreased $679 \mathrm{H} 3 \mathrm{~K} 9 \mathrm{me} 3$ post-differentiation only in the egg chambers but not in germarium (white dotted line). 680 (J) Quantification of H3K9me3 expression in the germline normalized to DAPI level in ovaries 681 depleted of SETDB1 (magenta) or wde (orange) compared to control ovaries (gray). Statistical 682 analysis was performed with Dunnett's multiple comparisons test; $N=10$ ovarioles; $n s=p>0.05$, $683 \quad{ }^{*}=p<0.05,{ }^{* *}=p<0.01,{ }^{* * *}=p<0.001$.

(K) Quantification of area of germarium and egg chambers during development in SETDB1 686 (magenta) or wde (orange) GKD ovaries compared to control ovaries (gray). Statistical analysis was performed with Dunnett's multiple comparisons test; $\mathrm{N}=10$ ovarioles; $n s=p>0.05,{ }^{*}=p<0.05$, $688{ }^{* *}=p<0.01,{ }^{* *}=p<0.001$. 

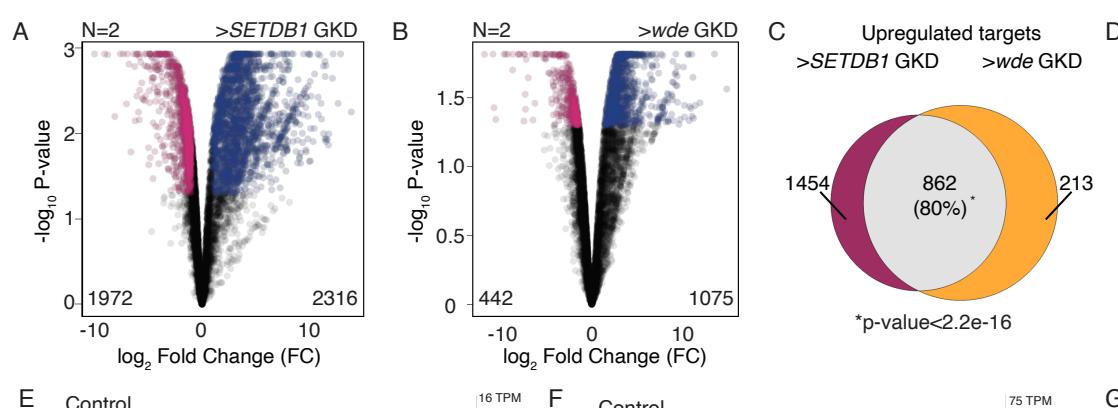

D Figure 2

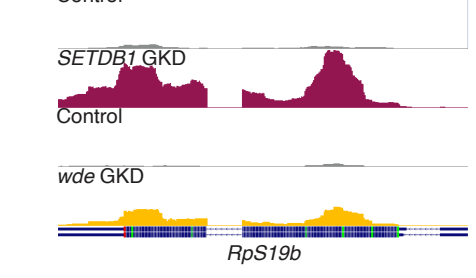

16 трм F Control
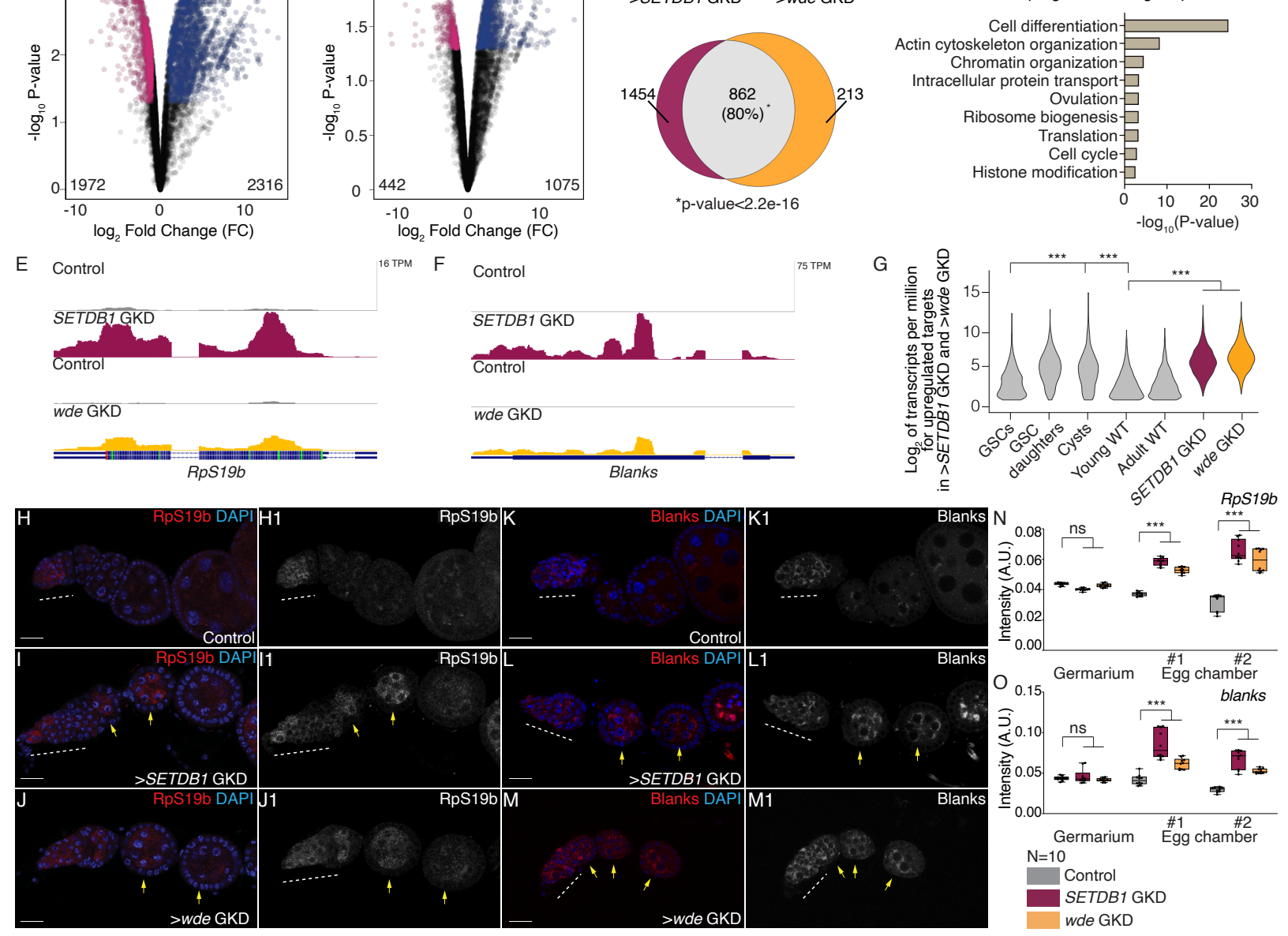

Figure 2: SETDB1/Wde represses a cohort of early oogenesis genes

(A-B) Volcano plots of - $\log _{10}$ P-value vs. Log 2 Fold Change (FC) of (A) SETDB1 and (B) wde GKD ovaries compared to control UAS-Dcr2;NG4NGT flies. Pink dots represent significantly downregulated transcripts and blue dots represent significantly upregulated transcripts in SETDB1, and wde GKD ovaries compared with control ovaries (FDR = False Discovery Rate < 0.05 and genes with 1.5 -fold or higher change were considered significant).

(C) Venn diagram of upregulated genes from RNA-seq of SETDB1 and wde GKD ovaries compared to UAS-Dcr2;NG4NGT. 862 targets are shared between GKD of SETDB1 and wde, suggesting that SETDB1 and Wde co-regulate a specific cohort of genes.

(D) The most significant biological process $G O$ terms of shared upregulated genes in ovaries depleted of SETDB1 and wde compared to UAS-Dcr2;NG4NGT control (FDR from p-values using a Fisher's exact test), showing differentiation as one of the significant processes regulated by 
713 (E-F) RNA-seq track showing that RpS19b and blanks is upregulated upon GKD of SETDB1 and 714 wde.

715

716 (G) Violin plot of mRNA levels of the 862 shared upregulated targets in ovaries enriched for GSCs, 717 cystoblasts, cysts, and whole ovaries, showing that the shared targets between SETDB1 and wde 718 are most highly expressed up to the cyst stages, that then tapers off in whole ovaries. Statistical 719 analysis performed with Hypergeometric test; ${ }^{* * *}$ indicates $p<0.001$.

(H-J1) Confocal images of germaria probed for RpS19b mRNA (red, grayscale) and DAPI (blue) in UAS-Dcr2;NG4NGT (H-H1) showing RpS19b RNA expression restricted to early stages of oogenesis and in GKD of SETDB1 (I-I1) and wde (J-J1) ovarioles showing RpS19b mRNA expression is expanded to egg chambers.

(K-M1) Confocal images of germaria probed for blanks mRNA (red, grayscale) and DAPI (blue) in UAS-Dcr2;NG4NGT (K-K1) showing blanks mRNA expression restricted to early stages of oogenesis and in GKD of SETDB1 (L-L1) and wde (M-M1) ovarioles where blanks mRNA expression is expanded to egg chambers.

(N-O) Quantification of fluorescence intensity of RpS19b (N) and blanks (O) mRNAs in the germarium and egg chambers during development in ovaries depleted of SETDB1 (magenta) or wde (orange) compared to control ovaries (gray). Statistical analysis was performed with Dunnett's multiple comparisons test; $\mathrm{N}=10$ ovarioles; $\mathrm{ns}=\mathrm{p}>0.05,{ }^{*}=\mathrm{p}<0.05,{ }^{* *}=\mathrm{p}<0.01,{ }^{* * *}=$ $735 \quad p<0.001$. 
bioRxiv preprint doi: https://doi.org/10.1101/2021.10.31.466575; this version posted November 2, 2021. The copyright holder for this preprint (which was not certified by peer review) is the author/funder, who has granted bioRxiv a license to display the preprint in perpetuity. It is made available under aCC-BY-NC-ND 4.0 International license.
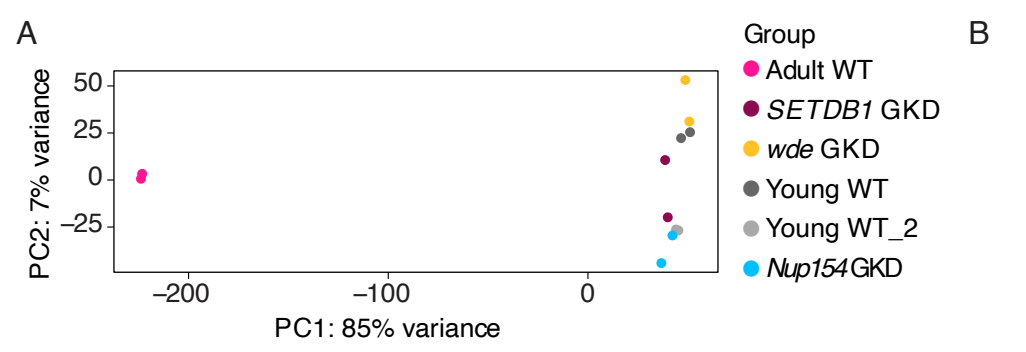

Figure S2
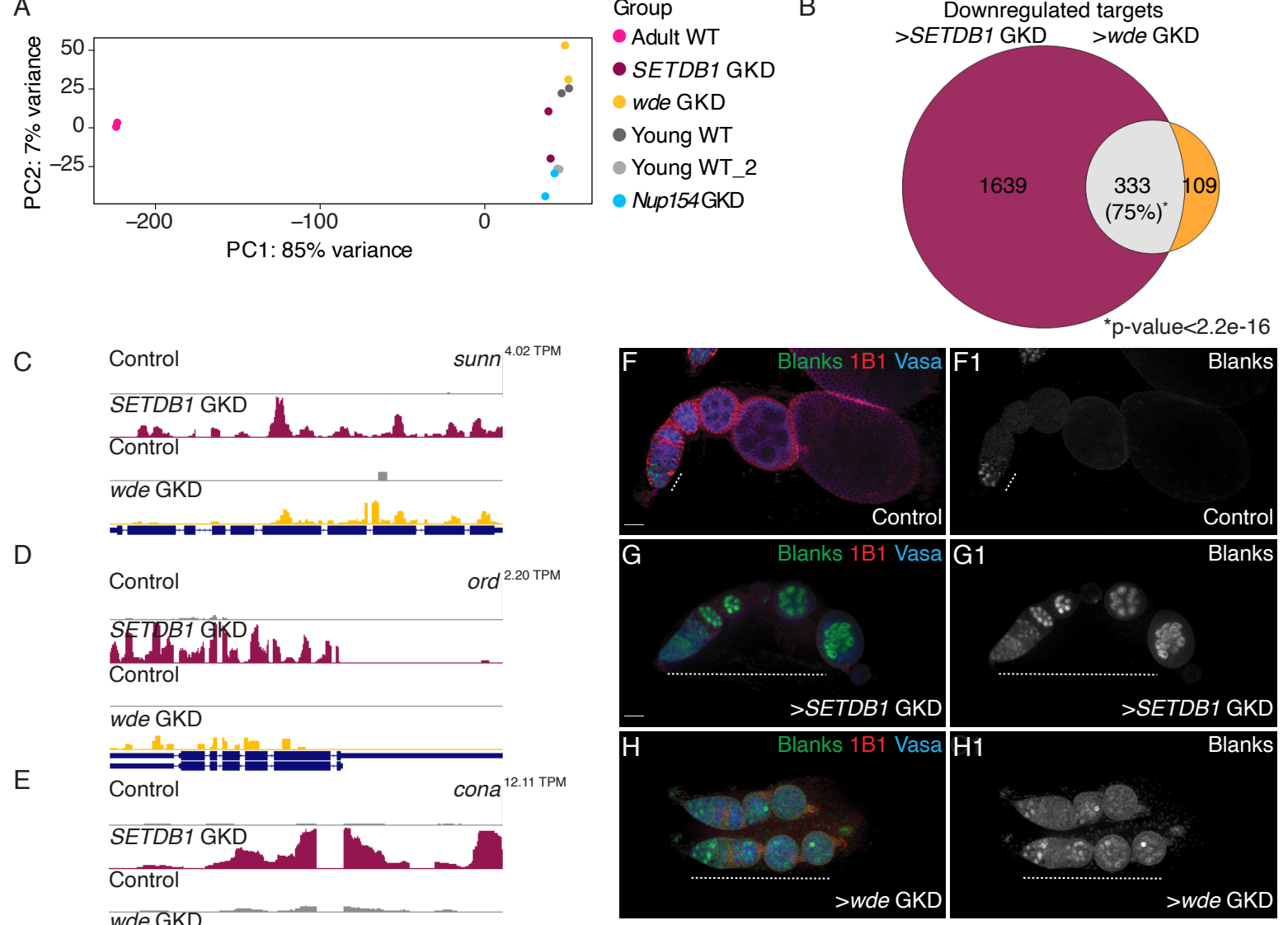

wde GKD

E
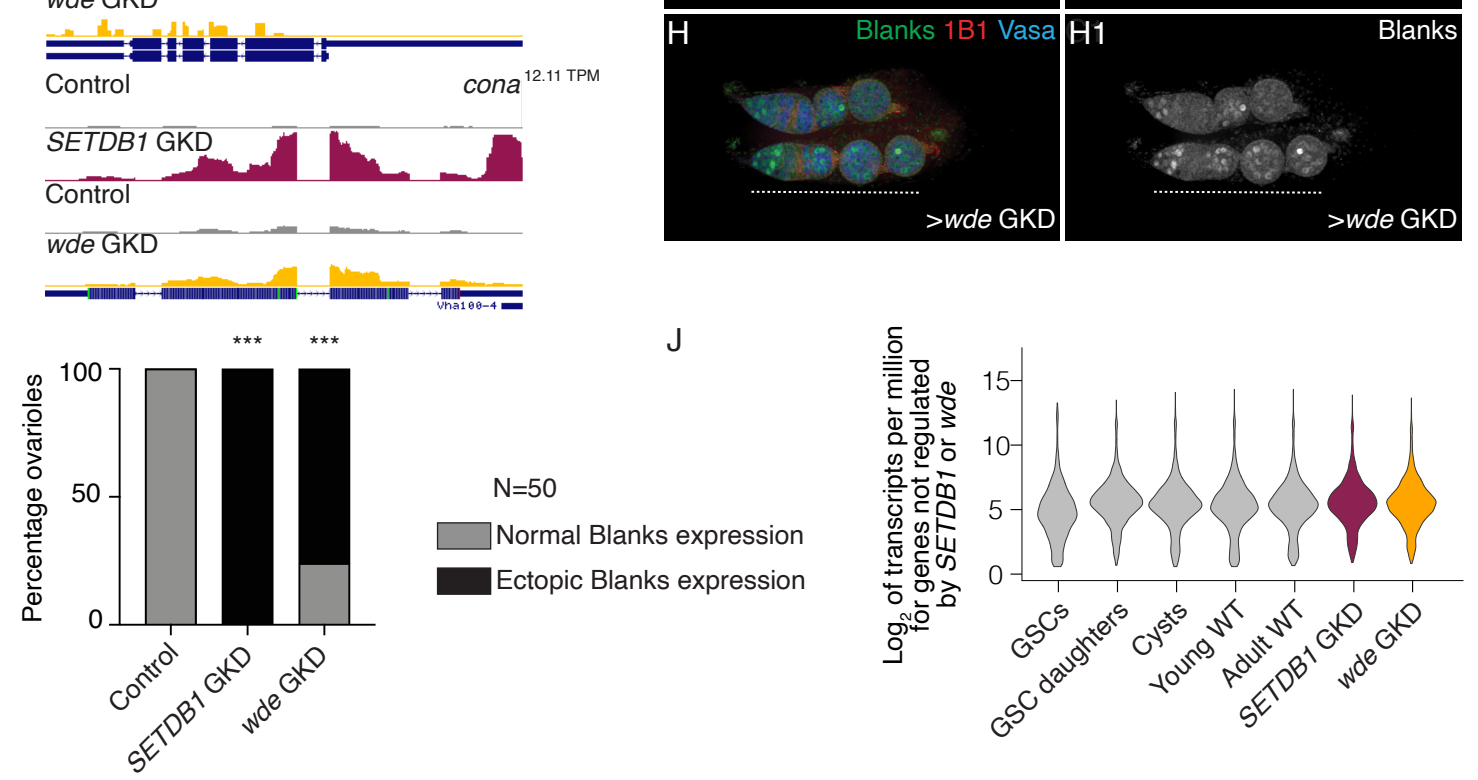

$\mathrm{N}=50$

Normal Blanks expression

Ectopic Blanks expression

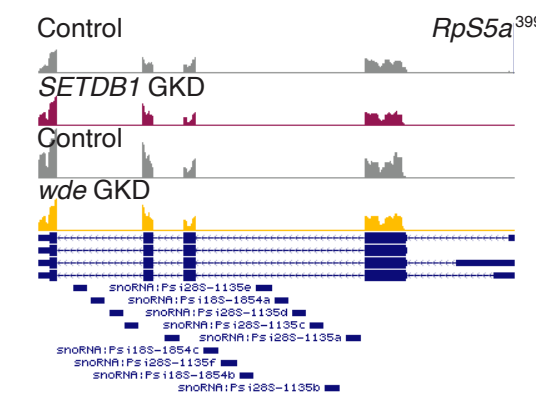

L

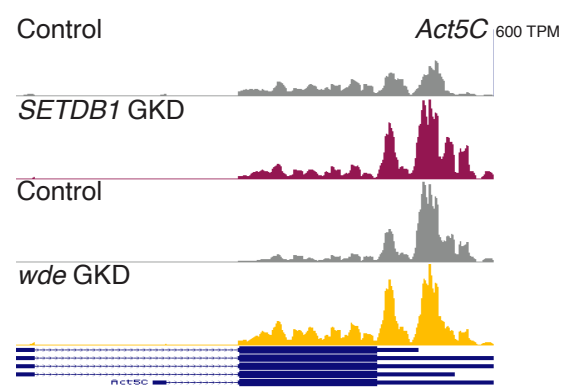


Supplementary Figure 2: SETDB1/Wde represses a cohort of genes that are broadly expressed prior to oocyte specification

742 (A) Principal Component Analysis (PCA) comparing RNA-seq data sets including adult WT, young

743 WT, SETDB1 GKD and wde GKD indicates that the SETDB1, wde and Nup154 GKD samples

744 are similar to young WT.

(B) Venn diagram of downregulated genes from RNA-seq of SETDB1 and wde GKD ovaries compared to UAS-Dcr2;NG4NGT. 333 targets are shared between SETDB1 and wde GKD.

(C-E) RNA-seq track showing that synaptonemal complex members sunn, ord and cona are upregulated upon SETDB1 and wde GKD.

(F-H1) Confocal images of UAS-Dcr2;NG4NGT (C-C1), SETDB1 (D-D1) and wde (E-E1) GKD ovarioles stained for 1B1 (red), Vasa (blue) and Blanks protein (green and grayscale) showing expanded Blanks expression in both SETDB1 and wde GKD egg chambers (arrow).

(I) Quantification of percentage ovarioles with ectopic Blanks expression (black) in SETDB1 or wde GKD ovaries to control ovaries ( $\mathrm{N}=50$ ovarioles; $100 \%$ in SETDB1 GKD and $82 \%$ in wde GKD compared to $0 \%$ in control.) Statistical analysis was performed with Fisher's exact test on ectopic Blanks expression; ${ }^{* * *}=p<0.001$.

(J) Violin plot of mRNA levels of the genes not regulated by SETDB1 or Wde in ovaries enriched for GSCs, cystoblasts, cysts, and whole ovaries, showing that SETDB1 and wde non-targets are not attenuated in the later egg chamber ovaries compared to earlier stages of oogenesis. 
A

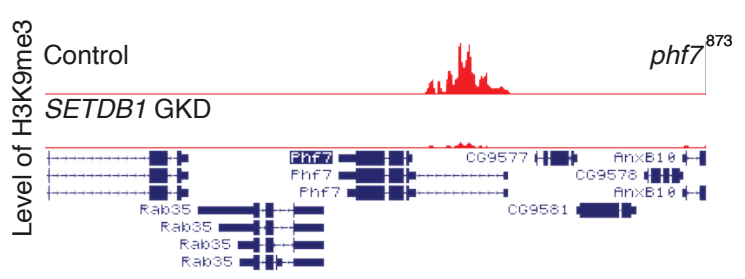

C

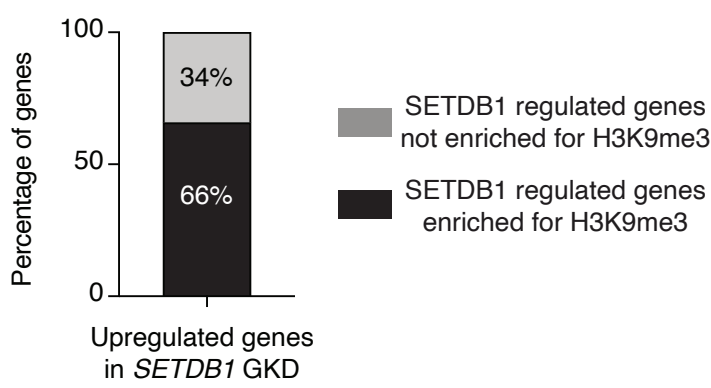

E

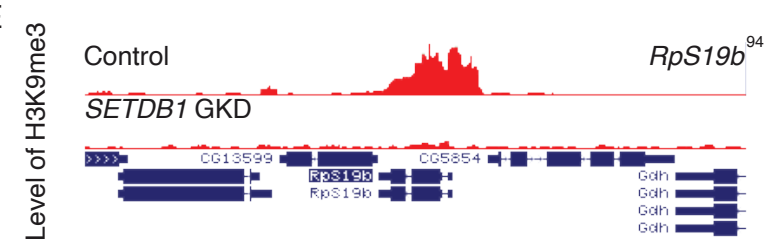

B

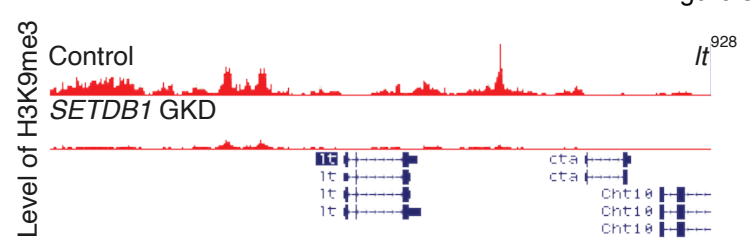

D

Genes that change $\mathrm{H} 3 \mathrm{~K} 9 \mathrm{me} 3$ enrichment upon SETDB1 GKD

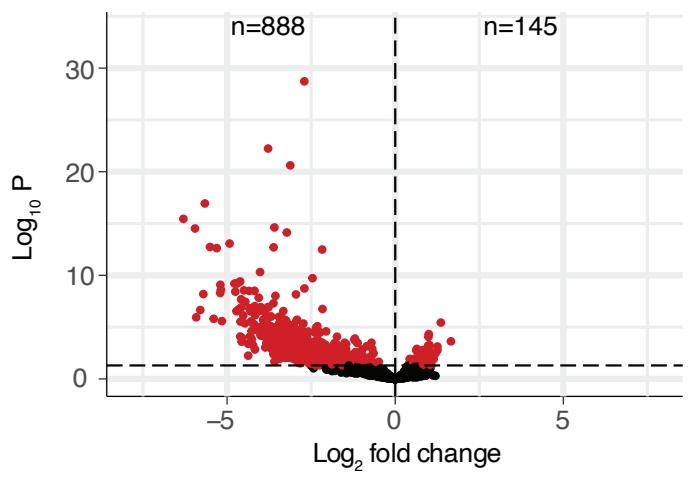

$\mathrm{F}$

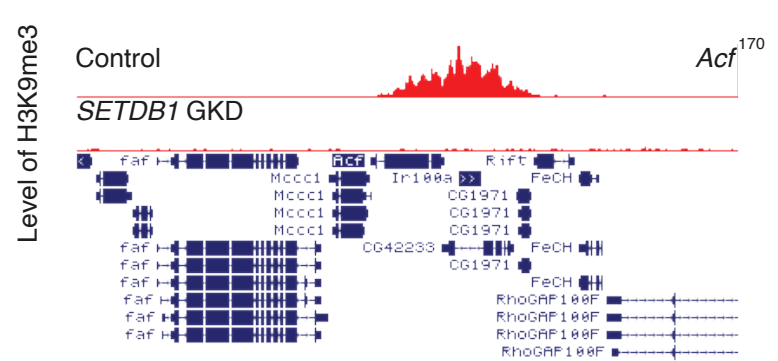

Figure 3: SETDB1 promotes silencing of early oogenesis genes by regulating levels of H3K9me3

(A-B) Tracks showing level of $\mathrm{H} 3 \mathrm{~K} 9 \mathrm{me} 3$ on previously validated and known heterochromatic genes phf7 and It respectively.

(C) Bar graph showing genes regulated by SETDB1 that are enriched for $\mathrm{H} 3 \mathrm{~K} 9 \mathrm{me} 3$ on the gene body. 1593 (black) out of 2316 (gray) genes upregulated upon loss of SETDB1 are enriched for H3K9me3.

(D) Volcano plot showing changes in H3K9me3 in SETDB1 GKD compared to WT. Genes that lose H3K9me3 are shown on the left (red). 888 genes lose H3K9me3 after SETDB1 GKD.

(E-F) Tracks showing level of H3K9me3 on target genes. Our data shows loss of H3K9me3 on SETDB1. 
A

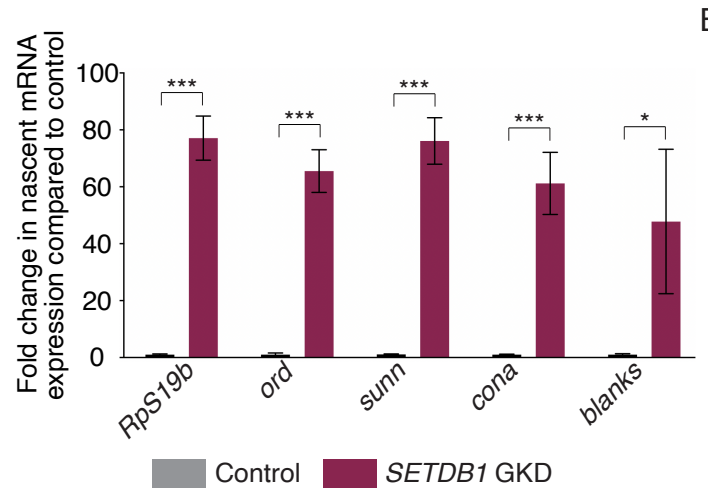

C

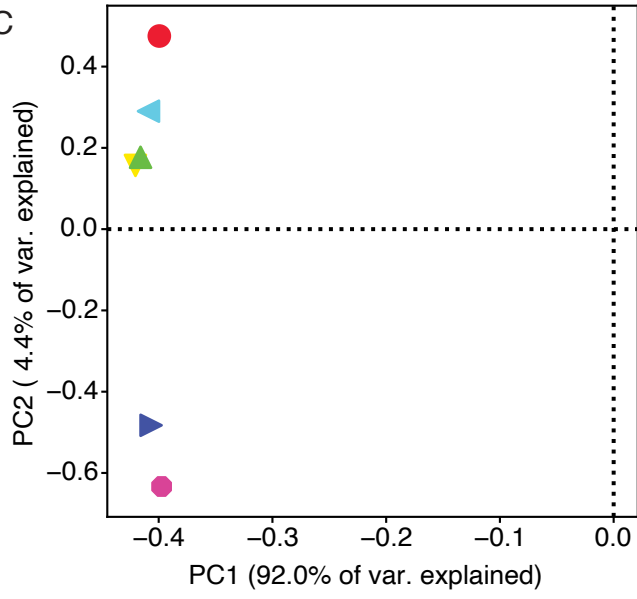

- $\triangle<$ Control

SETDB1 GKD
B
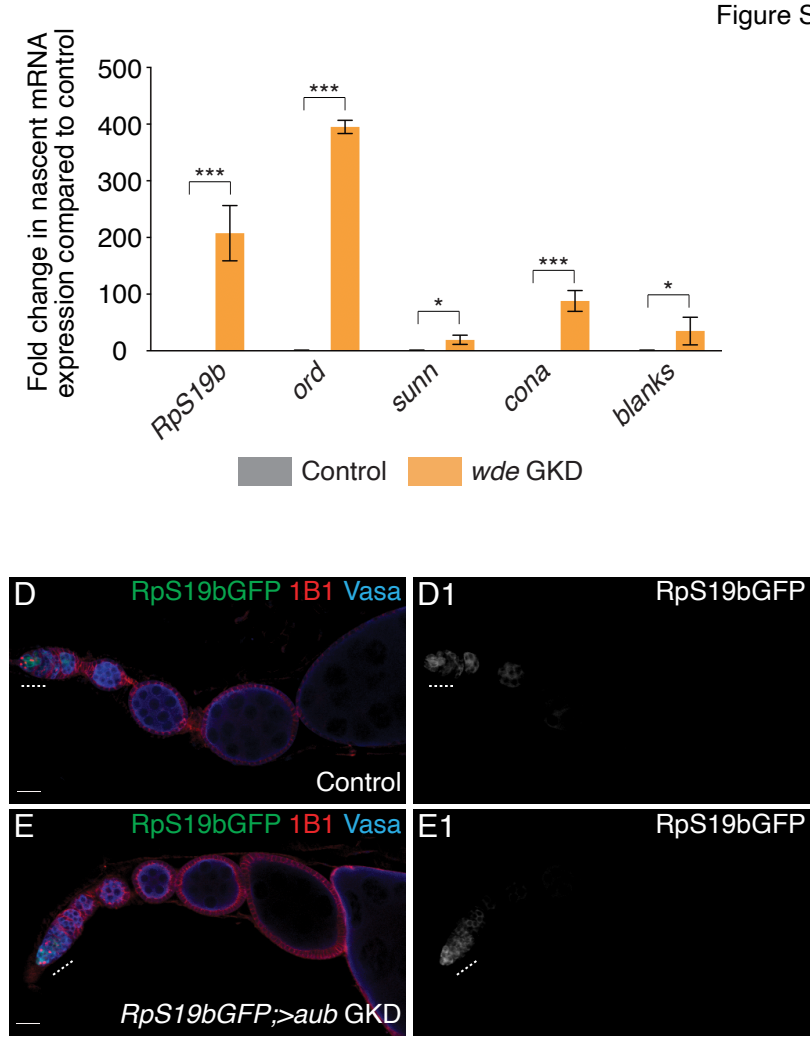

$\mathrm{F}$

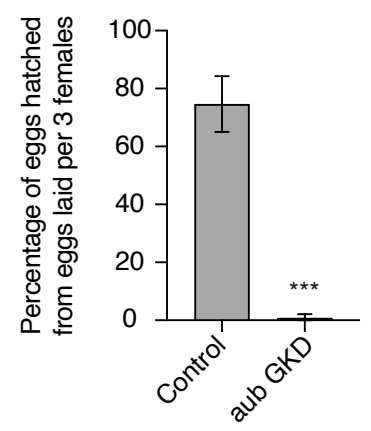

Figure S3 
800 genes are upregulated $\left(n=3,{ }^{*}=p \leq 0.05,{ }^{* *}=p<0.01,{ }^{* * *}=p<0.001\right.$, Error bars are SEM, 801 Student's t-Test).

802

803 (C) Principal Component Analysis (PCA) comparing CUT\&RUN data sets for control and SETDB1 804 GKD.

805

806 (D-E1) Ovariole from control RpS19b-GFP (D-D1) and GKD of aub (E-E1) stained for GFP (green, 807 right grayscale), Vasa (blue) and 1B1 (red). Depletion of this gene shows normal development of 808 the egg chambers and there was no ectopic expression of RpS19b-GFP in the egg chambers 809 suggesting SETDB1-mediated silencing of RpS19b-GFP is independent of piRNA pathway.

810

811 (F) Fertility assay of aub GKD indicating there was significant decrease in number of adult flies 812 that eclosed from the eggs laid by aub GKD flies compared to those from control flies ( $\mathrm{n}=3$ trials). $813^{* * *}=p<0.001$, Tukey's post-hoc test after one-way ANOVA.

814 
bioRxiv preprint doi: https://doi.org/10.1101/2021.10.31.466575; this version posted November 2, 2021. The copyright holder for this preprint (which was not certified by peer review) is the author/funder, who has granted bioRxiv a license to display the preprint in perpetuity. It is made available under aCC-BY-NC-ND 4.0 International license.

A

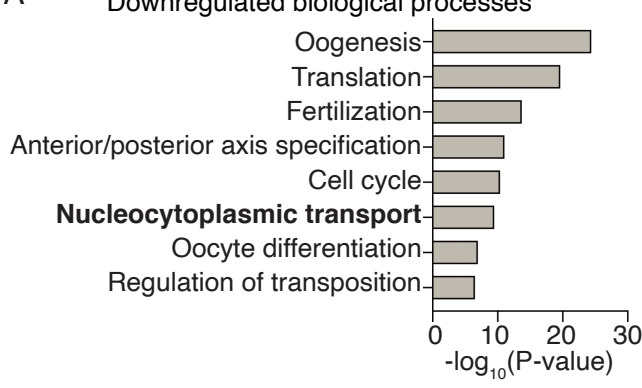

C

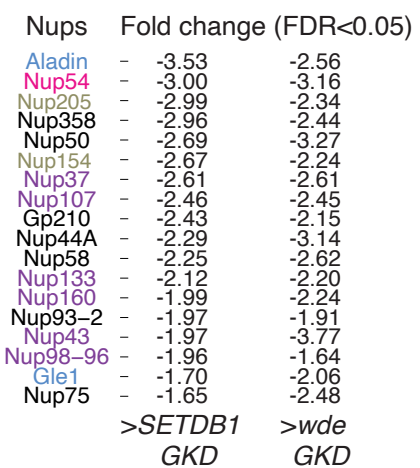

B
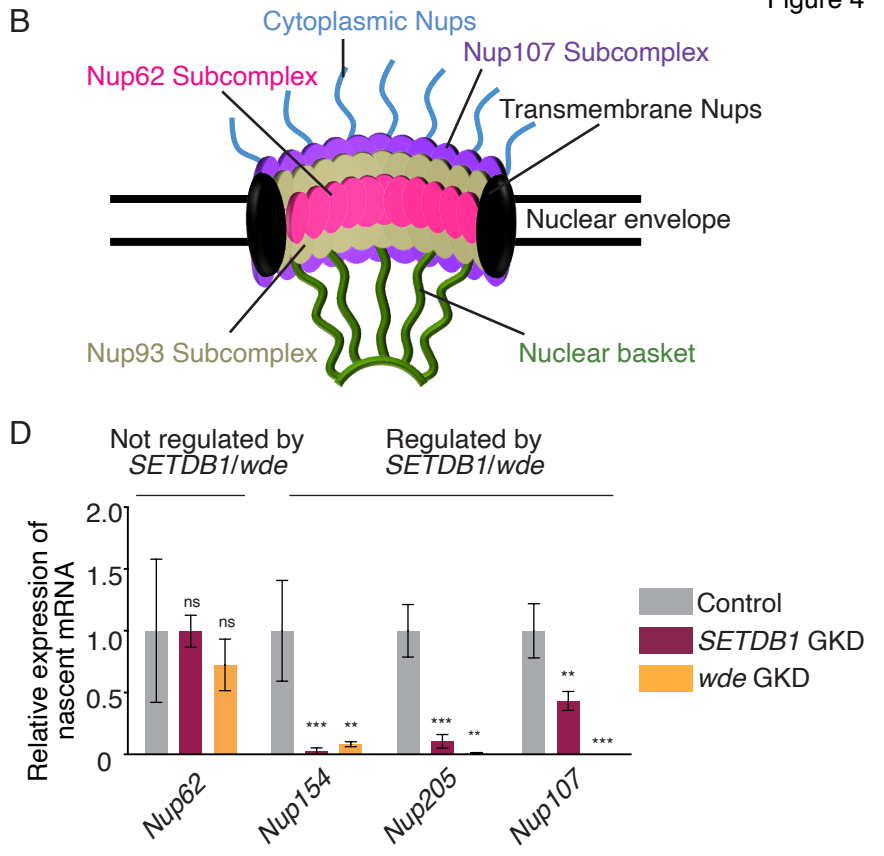
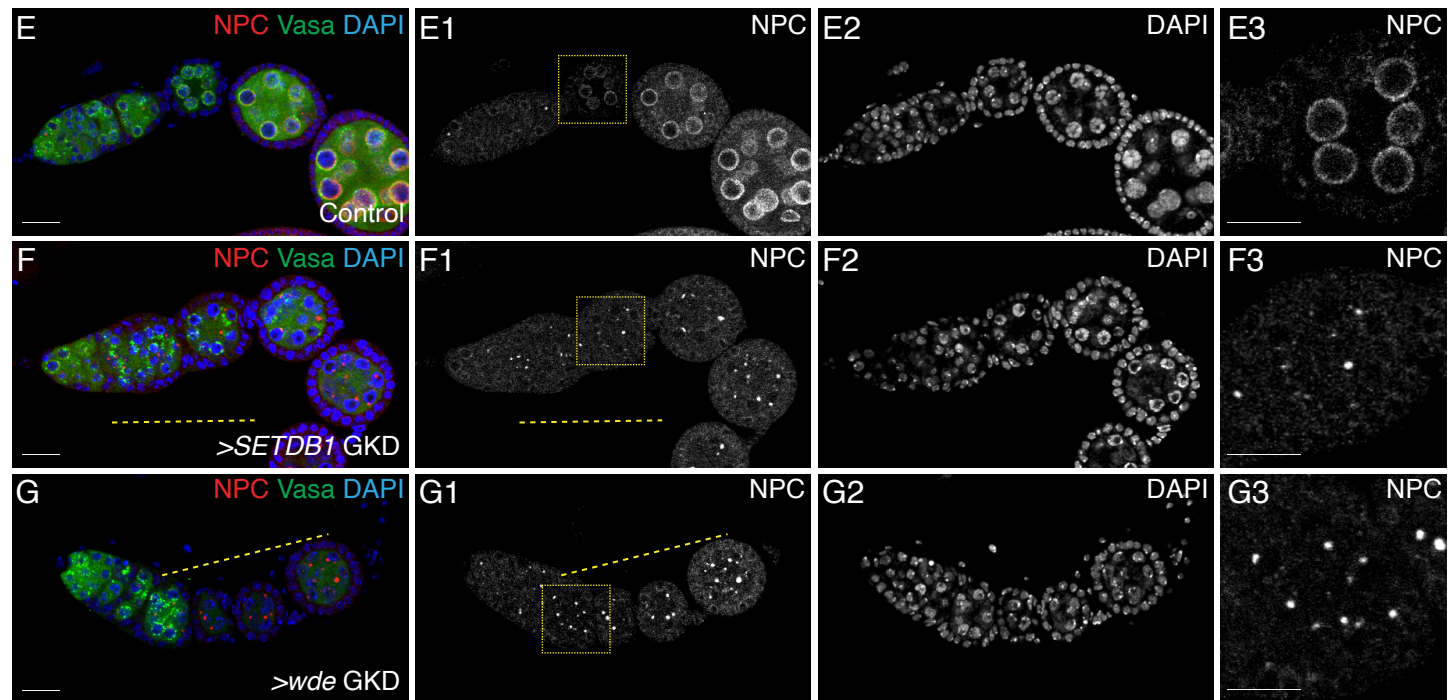

Figure 4
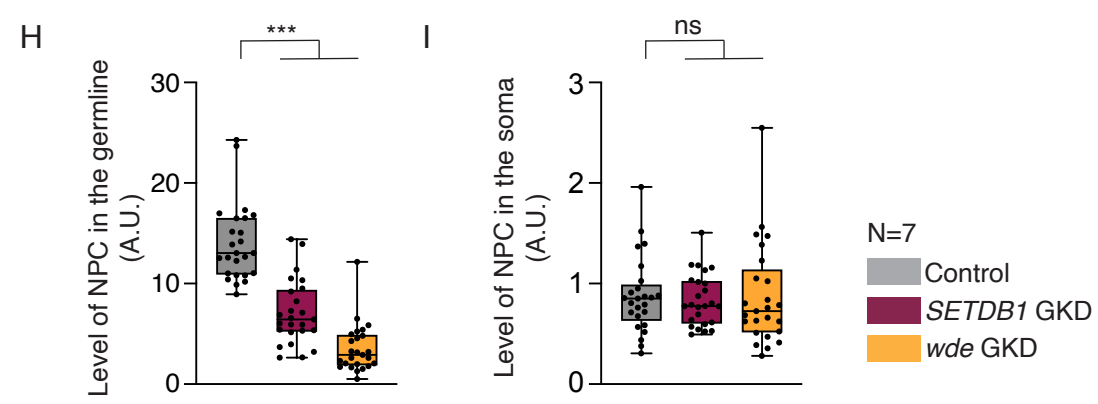
Figure 4: SETDB1/Wde promotes expression of a subset of nucleoporin genes and NPC formation

(A) The significant biological process GO terms of common downregulated genes in SETDB1 or wde GKD ovaries compared to UAS-Dcr2;NG4NGT control (FDR from p-values using a Fisher's exact test), showing nucleocytoplasmic transport as one of the significant processes regulated by SETDB1/Wde.

(B) A schematic of the Nucleopore Complex (NPC) showing cytoplasmic ring, nuclear ring and basket facing nucleoplasm and a central scaffold spanning the nuclear membrane. NPC is composed of several subcomplexes and $\sim 30$ nucleoporins (nups).

(C) Table showing levels of 18 nucleoporin mRNAs that are down regulated 1.5 or more fold in both SETDB1 or wde GKD ovaries compared to UAS-Dcr2;NG4NGT control ovaries.

(D) qRT-PCR assaying the pre-mRNA levels of SETDB1 and Wde-regulated Nup genes, including Nup154, Nup205 and Nup107 are decreased compared to control UAS-Dcr2;NG4NGT while levels of non-target Nup62 pre-mRNA is not affected (control level vs SETDB1 GKD and wde RNA in=3, ${ }^{* *}=p<0.01,{ }^{* *}=p<0.001$, Error bars are SEM, Student's t-Test).

(E-G3) Ovariole and egg chamber images of control UAS-Dcr2;NG4NGT (E-E3), GKD of SETDB1 (F-F3) and wde (G-G3) stained for NPC (red, grayscale), Vasa (green) and DAPI (blue). NPC staining was done using mab414 antibody. Depletion of SETDB1 shows reduced expression of NPC in the egg chambers suggesting SETDB1 regulates expression of several nucleoporins which in turn regulates formation of NPC.

$(\mathrm{H}-\mathrm{I})$ A.U. quantification of NPC level in the germline $(\mathrm{H})$ and soma $(\mathrm{I})$ in SETDB1 and wde GKD ovaries compared to UAS-Dcr2;NG4NGT control. Statistical analysis was performed with Dunnett's multiple comparisons test; $\mathrm{N}=25$ ovariole for germline and 15 for somatic quantitation; ns $=p>0.05,{ }^{*}=p \leq 0.05,{ }^{* *}=p<0.01,{ }^{* * *}=p<0.001$. 
bioRxiv preprint doi: https://doi.org/10.1101/2021.10.31.466575; this version posted November 2, 2021. The copyright holder for this preprint (which was not certified by peer review) is the author/funder, who has granted bioRxiv a license to display the preprint in perpetuity. It is made available under aCC-BY-NC-ND 4.0 International license.

Figure S4
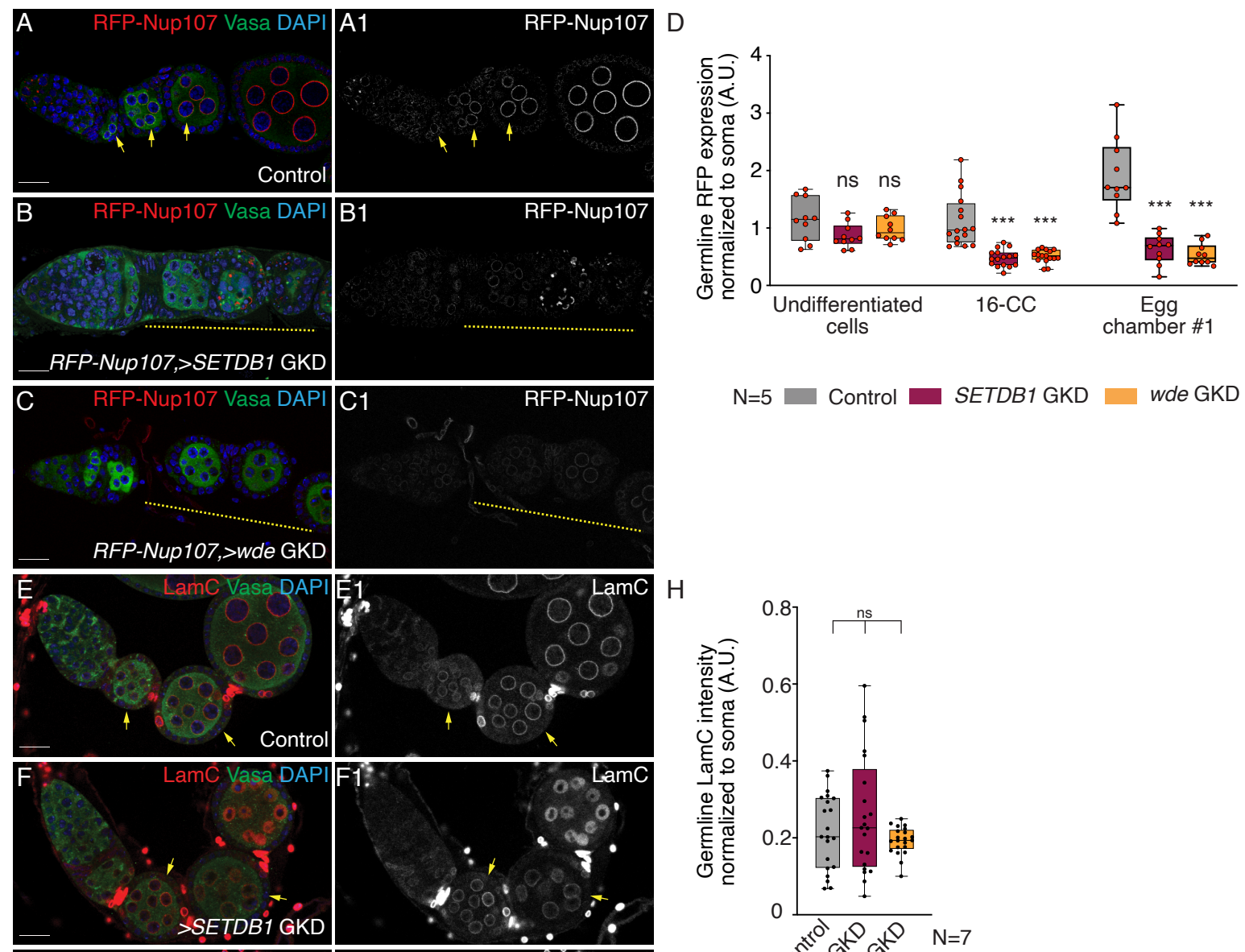

$\mathrm{H}$
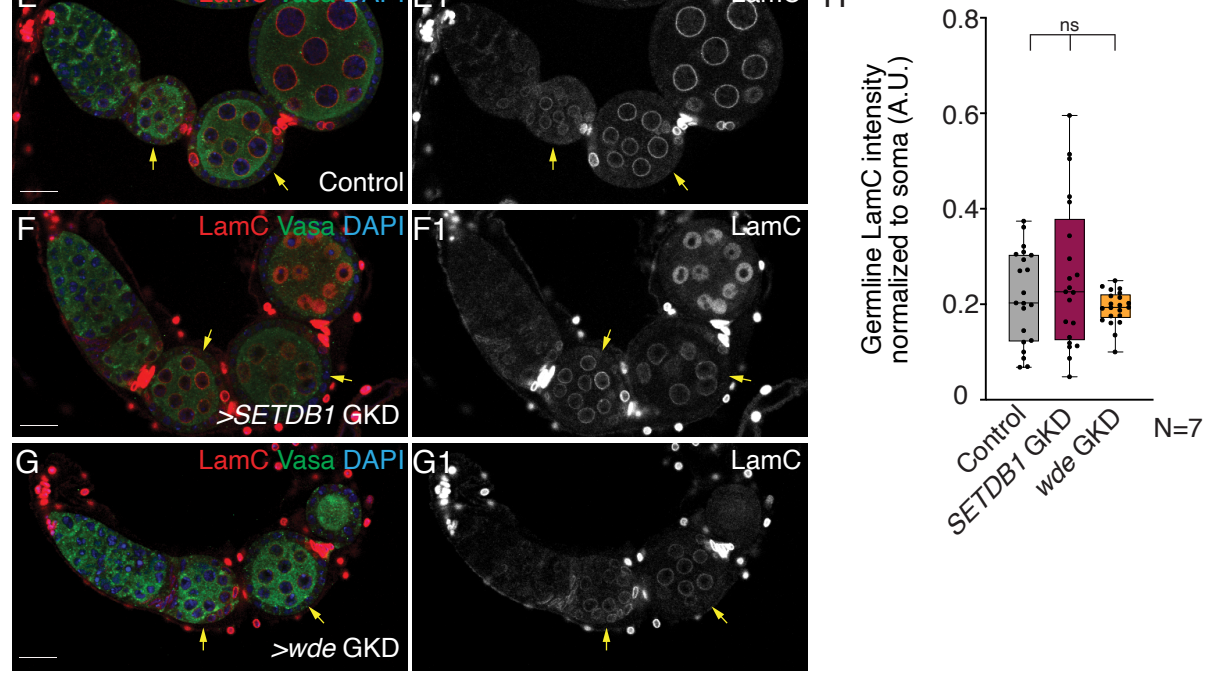
852 Supplementary Figure 4: SETDB1/Wde promotes NPC formation without affecting Lamin 853 C

(A-C1) Ovariole of control RFP-Nup107 A-A1), GKD of SETDB1 (B-B1) and wde (C-C1) stained 856 for RFP (red, right grayscale), Vasa (green) and DAPI (blue). Depletion of SETDB1 or wde shows 857 lower expression of RFP in the egg chambers (yellow line) suggesting SETDB1/wde regulates 858 expression of Nup107.

(D) A.U. quantification of RFP level in the germline normalized to soma in SETDB1- and wde861 GKD ovaries compared to control. Statistical analysis was performed with Dunnett's multiple comparisons test; $\mathrm{N}=5$ ovariole; $\mathrm{ns}=\mathrm{p}>0.05,{ }^{*}=\mathrm{p} \leq 0.05,{ }^{* *}=p<0.01,{ }^{* *}=p<0.001$.

(E-G1) Ovariole of control UAS-Dcr2;NG4NGT (E-E2), GKD of SETDB1 (F-F2) and wde (G-G2) stained for LamC (red, right grayscale), Vasa (green) and DAPI (blue). Depletion of SETDB1 or wde shows similar expression of LamC in the egg chambers suggesting SETDB1 or wde depletion does not affect expression of LamC.

(H) A.U. quantification of LamC level in the germline normalized to soma in SETDB1- and wdeGKD ovaries compared to UAS-Dcr2;NG4NGT control. Statistical analysis was performed with Dunnett's multiple comparisons test; $N=7$ ovariole; $n s=p>0.05$. 

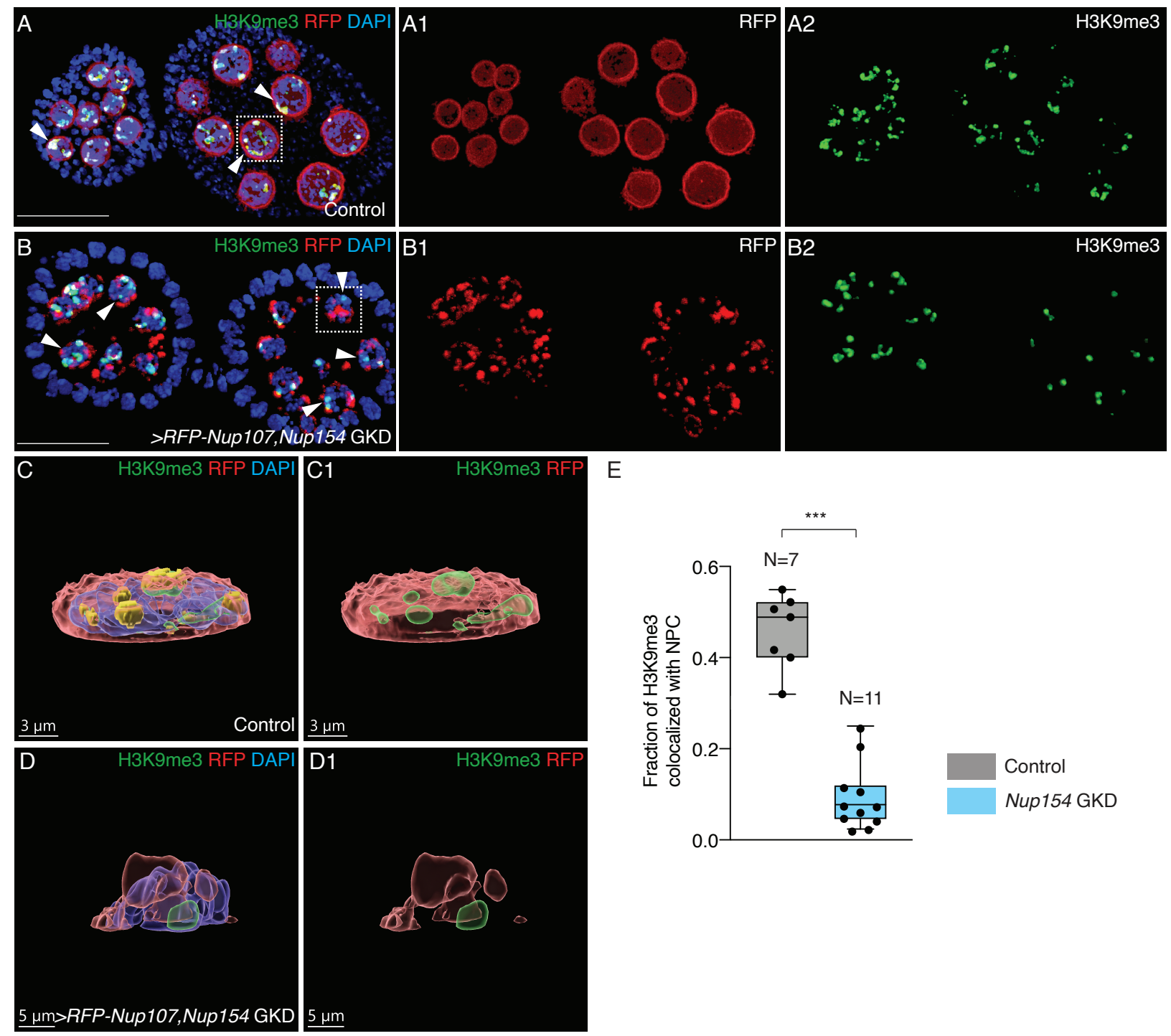

Figure 5: H3K9me3 heterochromatin colocalizes with NPC component Nup107 at the nuclear periphery

884 (A-A2) Egg chambers of control UAS-Dcr2;NG4NGT ovariole showing RFP-Nup107 (red, right red channel), H3K9me3 (green, right green channel). Heterochromatin is seen in close association with NPC (white arrows). Colocalized fraction is shown in yellow.

(B-B2) Egg chambers of Nup154 GKD ovariole showing significant decrease in the colocalization (white arrows) between RFP-Nup107 (red, right red channel) and H3K9me3 (green, right green 890 channel).

891 
894 and H3K9me3 (green). This shows that H3K9me3 heterochromatin domains (green) are formed 895 at the nuclear periphery and closely associate with Nup107.

896

897 (D-D1) 3D reconstruction of a single nuclei (white dotted box in B) from an egg chamber of 898 Nup154 GKD showing colocalization (yellow) of H3K9me3 with Nup107. This shows significant 899 reduction in colocalized fraction of H3K9me3 with Nup107.

900

(E) Quantification of levels of H3K9me3 that colocalizes with NPC in the germline of control ovarioles (gray) in contrast to Nup154 GKD ovarioles (blue). Quantitative object based colocalization was measured in Imaris software, ${ }^{* * *}=p<0.001$, one-tailed Students $t-T e s t$.

905

906

907 

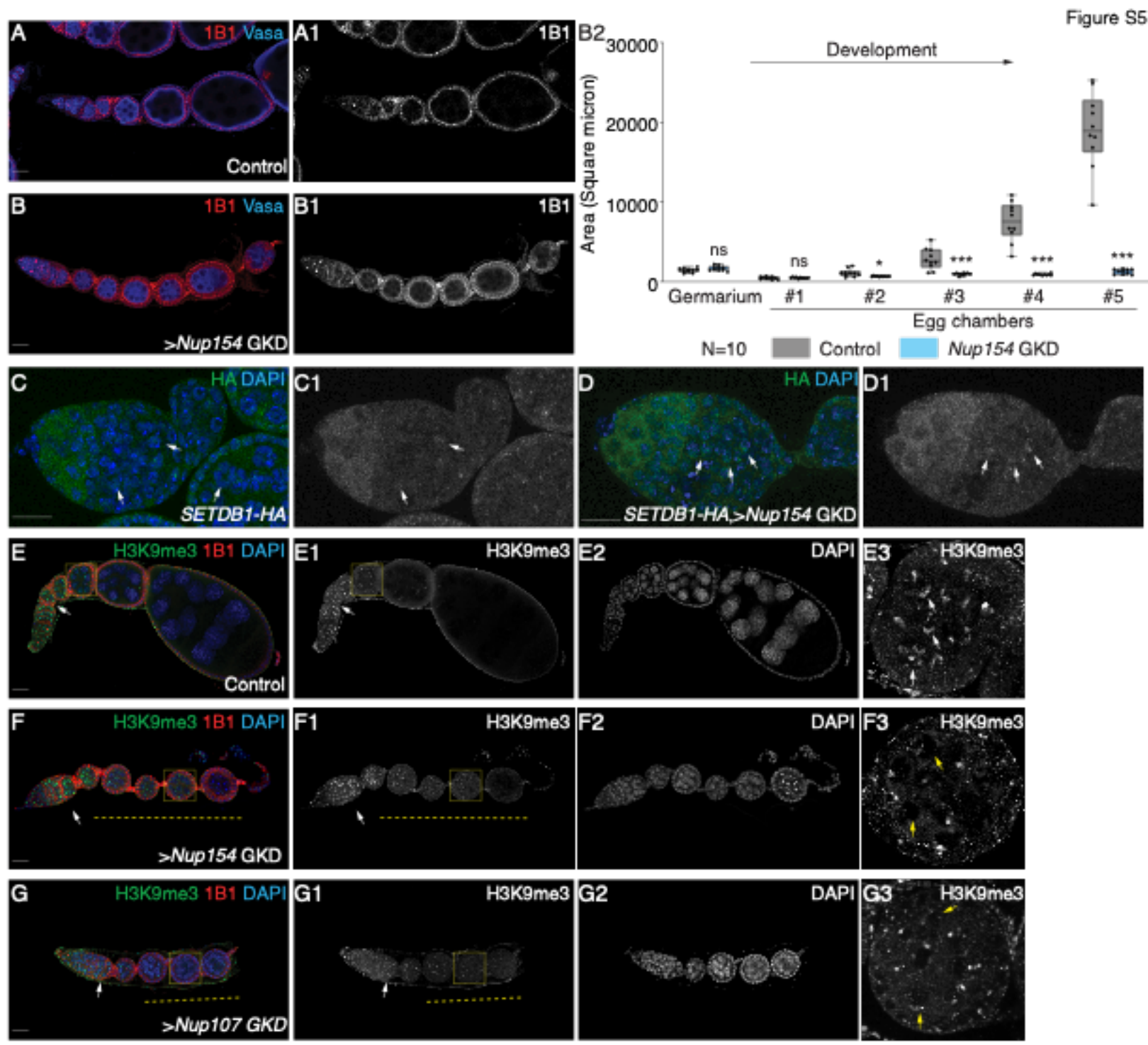

$\mathrm{H}$
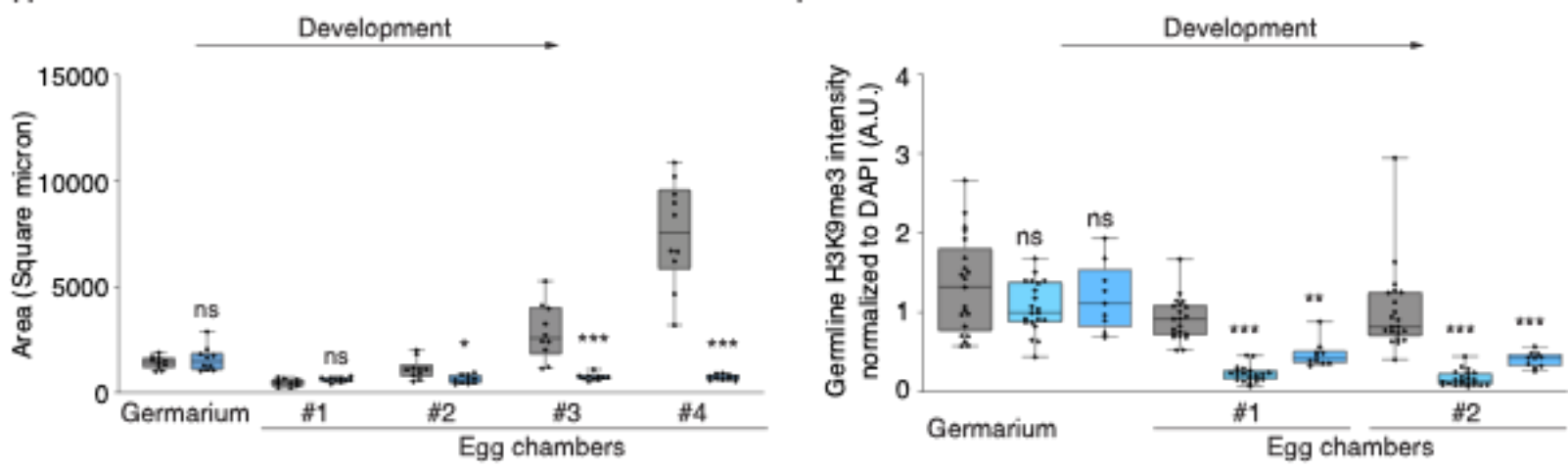

$\mathrm{N}=10 \quad$ Control Nup107 GKD

Control, N=7 Nup154 GKD, N=7 Nup107 GKD, N=5

\section{Supplementary Figure 5: NPC is required for maintaining heterochromatin}


911 (A-B2) Ovariole of control UAS-Dcr2;NG4NGT (A-A1) and GKD of Nup154 (B-B1) stained for 1B1

912 (red, right grayscale) and Vasa (blue). Control shows normal development of egg chambers while

913 Nup154 GKD shows egg chambers that do not grow. (B2) Quantification of area of germarium

914 and egg chambers during development in ovaries depleted of Nup154 (blue) compared to control

915 ovaries (gray). Statistical analysis was performed with Student's t-test; $\mathrm{N}=10$ ovarioles; $\mathrm{ns}=$

$916 \mathrm{p}>0.05,{ }^{*}=p \leq 0.05,{ }^{* *}=p<0.01,{ }^{* * *}=p<0.001$.

917

918 (C-D) Germaria of flies carrying HA tagged SETDB1 (C-C1) and GKD of Nup154 (D-D1) stained

919 for HA (green, right grayscale) and Vasa (blue). White arrows point at nuclear HA. Depletion of

920 germline Nup154 shows HA is present in the nucleus.

922 (E-G3) Ovariole and egg chamber of control UAS-Dcr2;NG4NGT (E-E3), GKD of Nup154 (F-F3)

923 and $\operatorname{Nup107}$ (G-G3) stained for H3K9me3 (green, right grayscale), DAPI (blue, right grayscale)

924 and 1B1 (red). Control shows H3K9me3 expression throughout oogenesis in the germline.

925 Depletion Nup154 and Nup107 results in decreased H3K9me3 (yellow dotted line) after

926 differentiation in the egg chambers. Late-stage egg chamber (yellow dotted squares) images

927 show decreased or loss of H3K9me3 in Nup154 and Nup107 GKD nurse cells (yellow arrows).

(H) Quantification of area of germarium and egg chambers during development in ovaries 930 depleted of Nup107 (blue) compared to control ovaries (gray). Statistical analysis was performed 931 with Student's t-test; $N=10$ ovarioles; $n s=p>0.05,{ }^{*}=p \leq 0.05,{ }^{* *}=p<0.01,{ }^{* * *}=p<0.001$.

(I) Quantification of H3K9me3 levels in the germline normalized to DAPI level in ovaries depleted of Nup154 (blue) and Nup107 (blue) compared to control ovaries (gray). Statistical analysis was performed with Dunnett's multiple comparisons test; ns $=p>0.05,{ }^{*}=p<0.05,{ }^{* *}=p<0.01,{ }^{* * *}=$ $936 \quad \mathrm{p}<0.001$. 
bioRxiv preprint doi: https://doi.org/10.1101/2021.10.31.466575; this version posted November 2, 2021. The copyright holder for this preprint (which was not certified by peer review) is the author/funder, who has granted bioRxiv a license to display the preprint in perpetuity. It is made available under aCC-BY-NC-ND 4.0 International license.

Figure 6

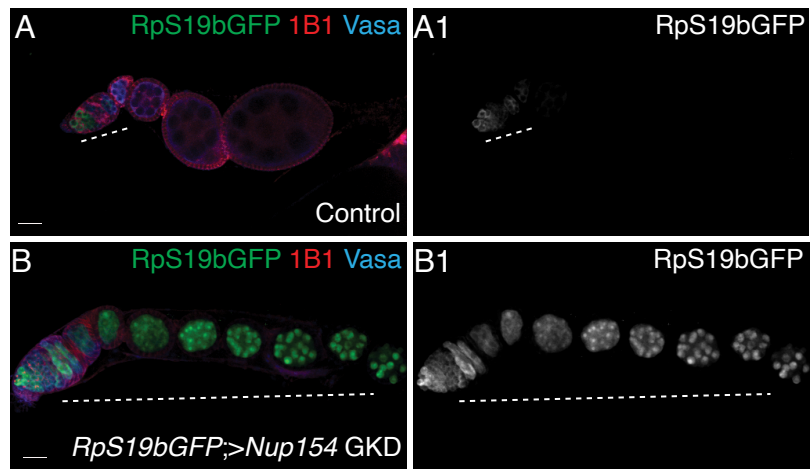

D

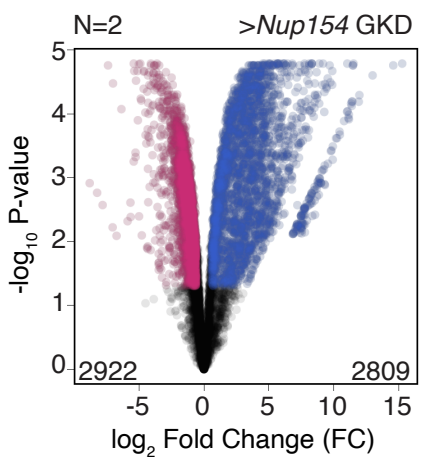

F

Control

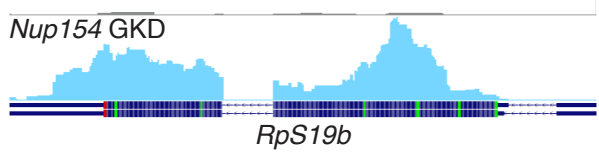

$\mathrm{H}$

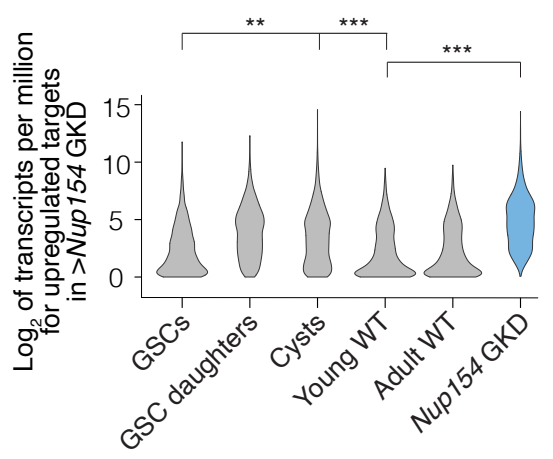

14.09 TPM

G

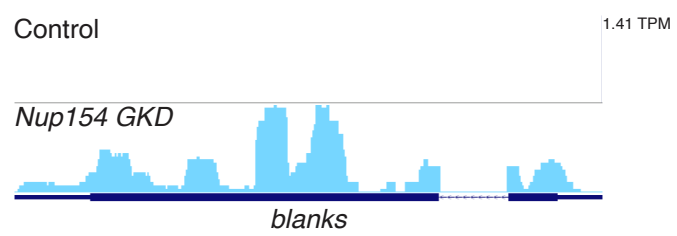

Genes directly silenced by SETDB1 and Nup154

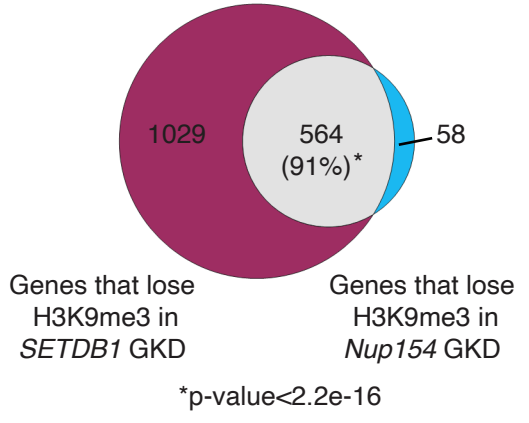

*p-value $<2.2 e-16$

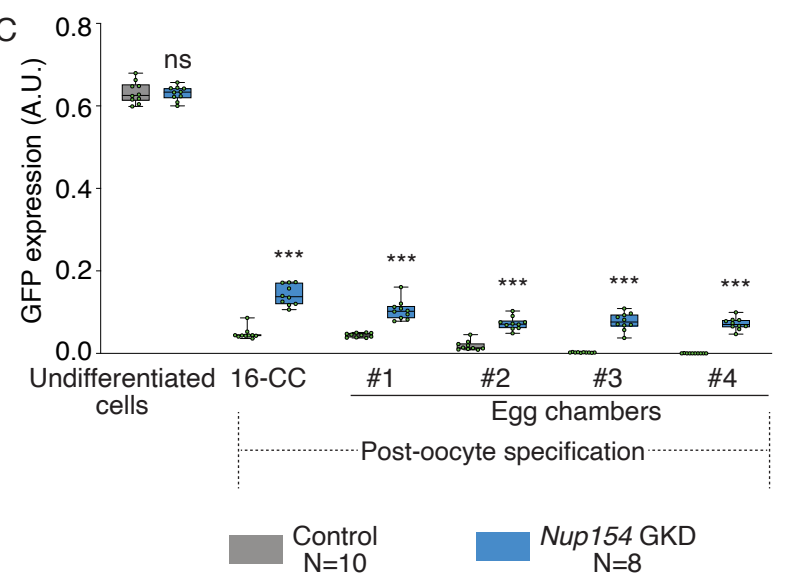

E

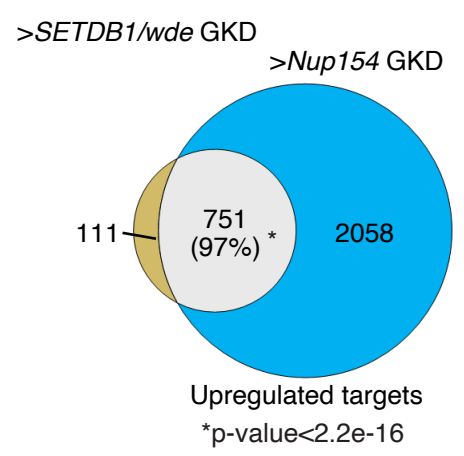


Figure 6: Nup154 is required for silencing a cohort of genes expressed during early oogenesis

(A-B1) Ovariole of control RpS19b-GFP (A-A1), GKD of Nup154 (B-B1) stained for GFP (green, right grayscale), Vasa (blue) and 1B1 (red). Depletion of Nup154 shows characteristic phenotype where the egg chambers did not grow and there was ectopic expression of RpS19b-GFP in the egg chambers (white dashed line).

(C) Arbitrary units (A.U.) quantification of RpS19b-GFP expression in the germarium and egg chambers during development upon GKD of Nup154 (blue) compared to control ovaries (gray). GFP is expressed higher in single cells in the germarium, decreases in the cyst stages, and then attenuated upon egg chamber formation in control. In Nup154 GKD, GFP expression persists in the egg chambers. Statistical analysis was performed with Dunnett's multiple comparisons test; $\mathrm{N}=10$ and 8 ovarioles for control and Nup154 GKD respectively; $\mathrm{ns}=\mathrm{p}>0.05,{ }^{*}=\mathrm{p}<0.05,{ }^{* *}=$ $p<0.01,{ }^{* *}=p<0.001$.

(D) Volcano plots of $-\log _{10} \mathrm{P}$-value vs. Log $_{2}$ Fold Change (FC) of mRNAs that show changes in Nup154 GKD compared to UAS-Dcr2;NG4NGT control ovaries. Pink dots represent significantly downregulated transcripts and blue dots represent significantly upregulated transcripts in Nup154 GKD ovaries compared with control ovaries (FDR = False Discovery Rate $<0.05$ and 1.5-fold or higher change were considered significant).

(E) Venn diagram of upregulated overlapping genes from RNA-seq of SETDB1 and wde and genes from Nup154 germline depleted ovaries compared to UAS-Dcr2;NG4NGT. 751 upregulated targets are shared between SETDB1, wde and Nup154 GKD, suggesting that Nup154 and SETDB1 function in co-regulating a specific set of genes.

(F-G) RNA-seq track showing that $R p S 19 b(\mathrm{~F})$ and blanks $(\mathrm{G})$ are upregulated upon germline depletion of Nup154.

(H) Violin plot of mRNA levels of the 2809 upregulated targets in ovaries enriched for GSCs, cystoblasts, cysts, and whole ovaries, showing that the upregulated targets of Nup154 are most highly enriched upto the cyst stages, and then tapers off in whole ovaries. Statistical analysis performed with Hypergeometric test; ${ }^{* * *}$ indicates $p<0.001$. and Nup154 in the germline. 622 genes lose H3K9me3 after Nup154 GKD out of which 564 genes are also directly silenced by SETDB1, suggesting co-regulation of these genes by both SETDB1 and Nup154. 
bioRxiv preprint doi: https://doi.org/10.1101/2021.10.31.466575; this version posted November 2, 2021. The copyright holder for this preprint (which was not certified by peer review) is the author/funder, who has granted bioRxiv a license to display the preprint in perpetuity. It is made available under aCC-BY-NC-ND 4.0 International license.
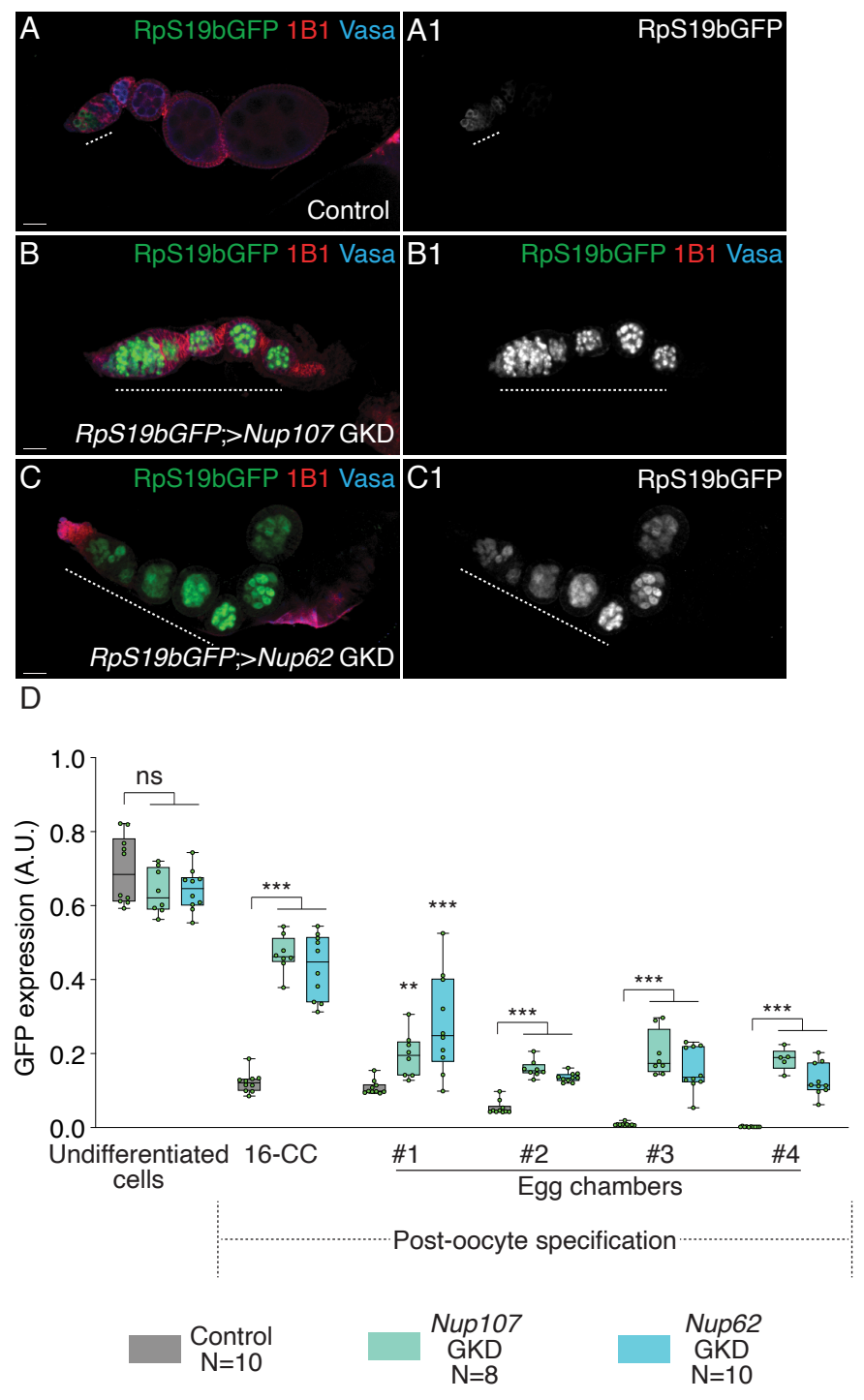

E $\quad>S E T D B 1 /$ wde GKD

$>$ Nup154 GKD

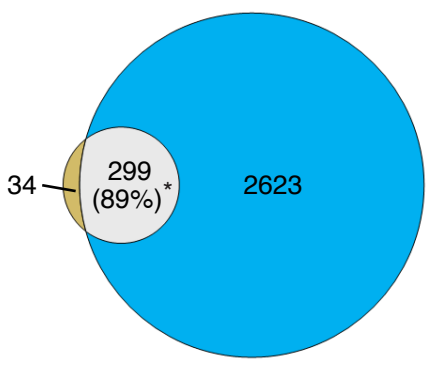

Downregulated targets

${ }^{*} p$-value $<2.2 e-16$
F Control

Figure S6

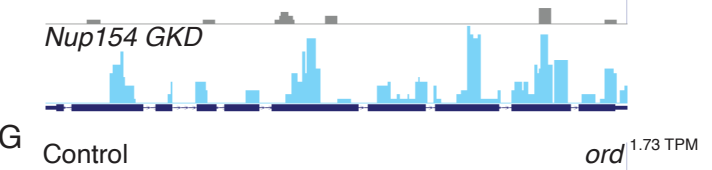

G

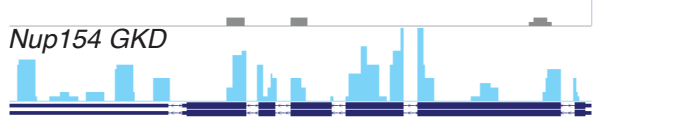

H Control

cona ${ }^{11.93 \text { TPM }}$

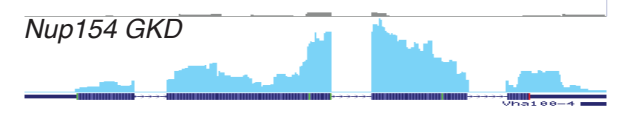

I$$
\text { 을 }
$$

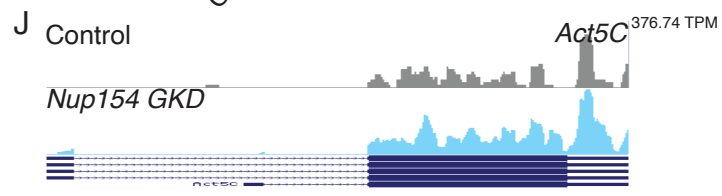

K

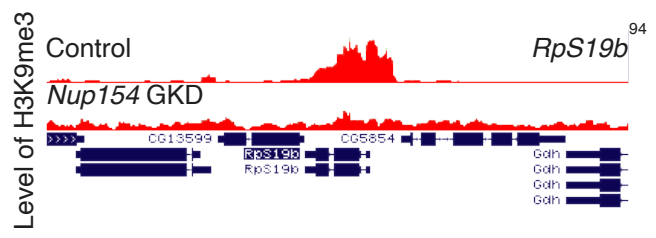

$\mathrm{L}$

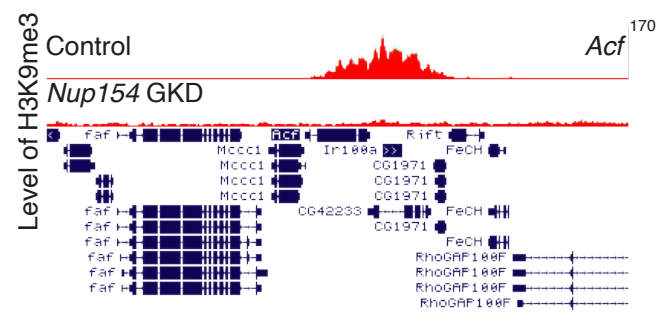


(A-C1) Ovariole from control RpS19b-GFP (A-A1), GKD of Nup107 (B-B1) and Nup62 (C-C1) stained for GFP (green, right grayscale), Vasa (blue) and 1B1 (red). Depletion of these Nups shows characteristic phenotype where the egg chambers do not grow and there is ectopic expression of RpS19b-GFP in the egg chambers (white dashed line).

(D) A.U. quantification of ectopic RpS19b-GFP expression in the germarium and egg chambers with development in ovaries of Nup107 (teal) and Nup62 (light blue) GKD compared to control ovaries (gray). Statistical analysis was performed with Dunnett's multiple comparisons test; $\mathrm{ns}=$ $p>0.05,{ }^{* *}=p<0.01,{ }^{* * *}=p<0.001$.

(E) Venn diagram of down regulated overlapping genes from RNA-seq of SETDB1 and wde regulated genes with Nup154 GKD ovaries compared to UAS-Dcr2;NG4NGT. 299 down regulated targets are shared between SETDB1, wde and Nup154 GKD, suggesting that Nup154 and SETDB1 function in co-regulating a specific set of genes.

1004

(F-H) RNA-seq track showing that synaptonemal complex members sunn, ord and cona are upregulated upon germline depletion of Nup154.

(I) Violin plot of mRNA levels of the genes not regulated by Nup154 in ovaries enriched for GSCs, cystoblasts, cysts, and young and adult whole ovaries, showing that the non-targets of Nup154 are not silenced in the ovaries compared to cyst stages and whole ovaries. Statistical analysis performed with Hypergeometric test; ${ }^{* * *}$ indicates $p<0.001$.

(J) RNA-seq track showing that upon germline depletion of Nup154, Act5C is unaffected. 
bioRxiv preprint doi: https://doi.org/10.1101/2021.10.31.466575; this version posted November 2, 2021. The copyright holder for this preprint (which was not certified by peer review) is the author/funder, who has granted bioRxiv a license to display the preprint in perpetuity. It is made available under aCC-BY-NC-ND 4.0 International license.

Figure 7
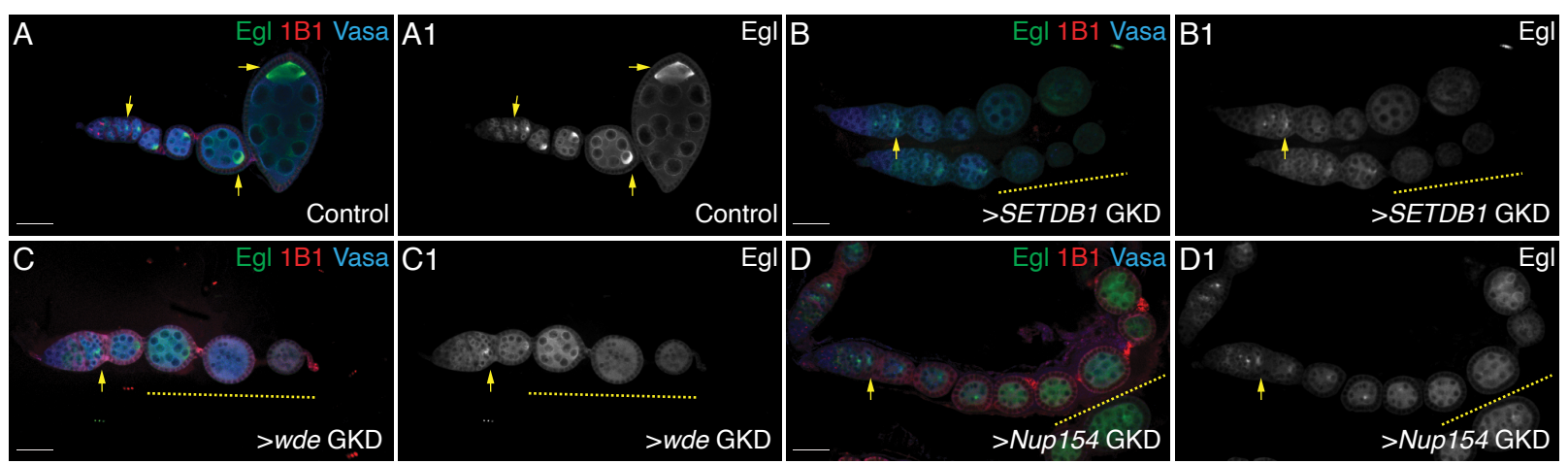

E

F
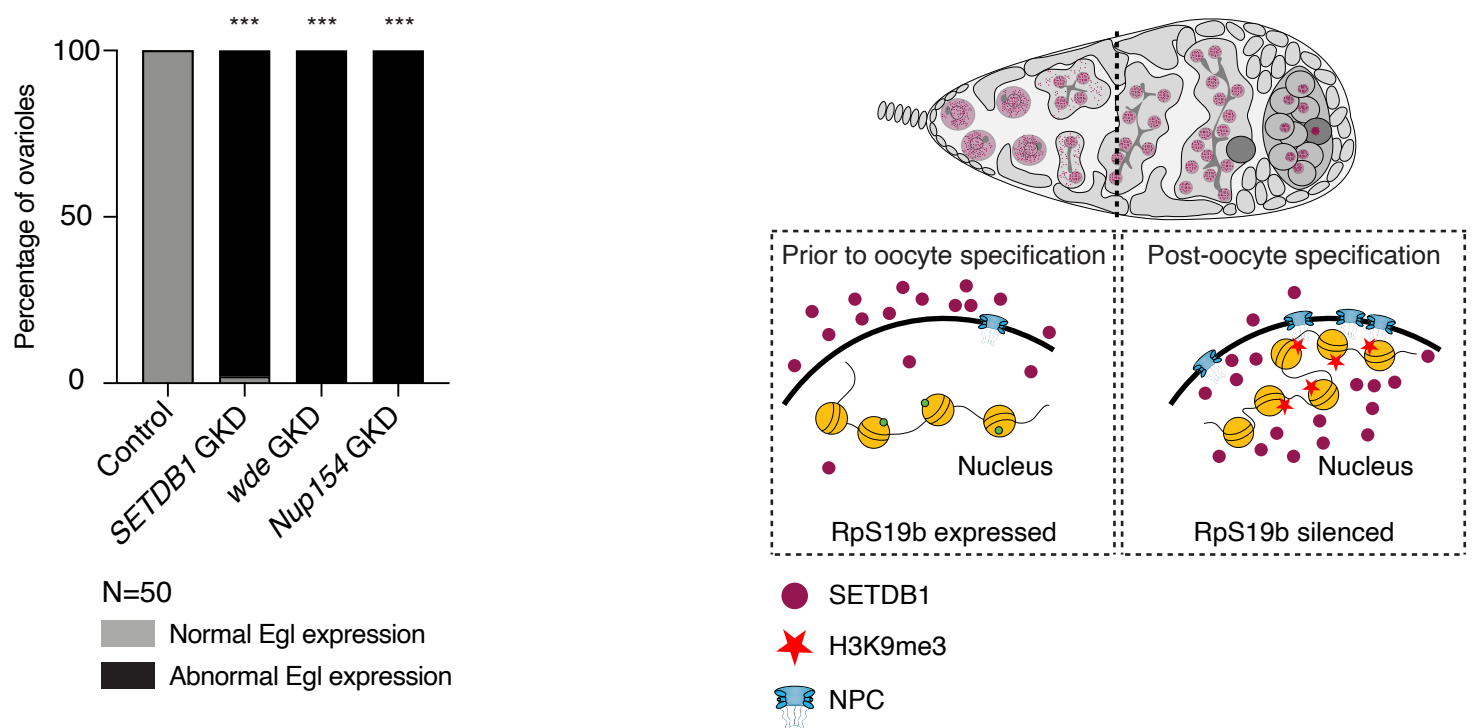
1022 Figure 7: Silencing of early oogenesis genes mediated by SETDB1, Wde and Nup154 is required for maintenance of oocyte fate

(A-D1) Ovarioles of control UAS-Dcr2;NG4NGT (A-A1), GKD of SETDB1 (B-B1), wde (C-C1) and Nup154 (D-D2) stained for Egl (green, right grayscale), Vasa (blue) and 1B1 (red). Control shows proper oocyte specification with one oocyte in each egg chamber. Depletion of SETDB1, wde and Nup154 in the germline results in initial oocyte specification (yellow arrow) which is then lost in the subsequent egg chambers (yellow dashed line).

(E) Quantification of percentage ovarioles with abnormal/loss of Egl expression (black) in ovaries depleted of SETDB1 or wde or Nup154 compared to control ovaries (gray) (N=50 ovarioles; $98 \%$ in SETDB1 GKD and 100\% in wde and Nup154 GKD compared to 0\% in control.) Statistical analysis was performed with Fisher's exact ${ }^{* * *}=p<0.001$.

(F) A model showing that nuclear translocation of SETDB1 after differentiation promotes heterochromatin formation mediated by deposition of H3K9me3 mark. This heterochromatin promotes increased NPC formation which then helps maintain heterochromatin. 


\section{References}

1042

1043

1044

1045

1046

1047

1048

1049

1050

1051

1052

1053

1054

1055

1056

1057

1058

1059

1060

1061

1062

1063

1064

1065

1066

1067

1068

1069

1070

1071

1072

1073

1074

1075

1076

1077

1078

1079

1080

1081

1082

1083

1084

1085

1086

1087

1088

1089

1090

Ables, E.T., 2015. Drosophila Oocytes as a Model for Understanding Meiosis: An Educational Primer to Accompany "Corolla Is a Novel Protein That Contributes to the Architecture of the Synaptonemal Complex of Drosophila." Genetics 199, 17-23. https://doi.org/10.1534/genetics.114.167940

Ahmad, K., 2018. CUT\&RUN with Drosophila tissues. https://doi.org/10.17504/protocols.io.umfeu3n

Andersen, P.R., Tirian, L., Vunjak, M., Brennecke, J., 2017. A heterochromatin-dependent transcription machinery drives piRNA expression. Nature. https://doi.org/10.1038/nature23482

Ben-Ami, F., Heller, J., 2005. Spatial and temporal patterns of parthenogenesis and parasitism in the freshwater snail Melanoides tuberculata. Journal of Evolutionary Biology 18, 138146. https://doi.org/10.1111/j.1420-9101.2004.00791.x

Blatt, P., Martin, E.T., Breznak, S.M., Rangan, P., 2020. Post-transcriptional gene regulation regulates germline stem cell to oocyte transition during Drosophila oogenesis. Curr Top Dev Biol 140, 3-34. https://doi.org/10.1016/bs.ctdb.2019.10.003

Blatt, P., Wong-Deyrup, S.W., McCarthy, A., Breznak, S., Hurton, M.D., Upadhyay, M., Bennink, B., Camacho, J., Lee, M.T., Rangan, P., 2021. RNA degradation is required for the germ-cell to maternal transition in Drosophila. Current Biology 31, 2984-2994.e7. https://doi.org/10.1016/j.cub.2021.04.052

Brickner, D.G., Randise-Hinchliff, C., Lebrun Corbin, M., Liang, J.M., Kim, S., Sump, B., D’Urso, A., Kim, S.H., Satomura, A., Schmit, H., Coukos, R., Hwang, S., Watson, R., Brickner, J.H., 2019. The Role of Transcription Factors and Nuclear Pore Proteins in Controlling the Spatial Organization of the Yeast Genome. Developmental Cell 49, 936-947.e4. https://doi.org/10.1016/j.devcel.2019.05.023

Cahoon, C.K., Hawley, R.S., 2016. Regulating the construction and demolition of the synaptonemal complex, Nature Structural and Molecular Biology. https://doi.org/10.1038/nsmb.3208

Calvi, B.R., Lilly, M.A., Spradling, A.C., 1998. Cell cycle control of chorion gene amplification. Genes Dev 12, 734-744. https://doi.org/10.1101/gad.12.5.734

Capelson, M., Doucet, C., Hetzer, M.W., 2010. Nuclear pore complexes: guardians of the nuclear genome. Cold Spring Harb Symp Quant Biol 75, 585-597. https://doi.org/10.1101/sqb.2010.75.059

Capelson, M., Hetzer, M.W., 2009. The role of nuclear pores in gene regulation, development and disease. EMBO Rep 10, 697-705. https://doi.org/10.1038/embor.2009.147

Capelson, Maya, Liang, Y., Schulte, R., Mair, W., Wagner, U., Hetzer, M.W., 2010. Chromatinbound nuclear pore components regulate gene expression in higher eukaryotes. Cell 140, 372-383. https://doi.org/10.1016/j.cell.2009.12.054

Carreira-Rosario, A., Bhargava, V., Hillebrand, J., Kollipara, R.K., Ramaswami, M., Buszczak, M., 2016. Repression of Pumilio Protein Expression by Rbfox1 Promotes Germ Cell Differentiation. Developmental Cell 36, 562-571.

Chen, D., McKearin, D., 2003a. Dpp signaling silences bam transcription directly to establish asymmetric divisions of germline stem cells. Current biology: CB 13, 1786-1791.

Chen, D., McKearin, D.M., 2003b. A discrete transcriptional silencer in the bam gene determines asymmetric division of the Drosophila germline stem cell. Development (Cambridge, England) 130, 1159-1170.

Chen, Y., Pane, A., Schüpbach, T., 2007. Cutoff and Aubergine mutations result in upregulation of retrotransposons and activation of a checkpoint in the Drosophila germline. Curr Biol 17, 637-642. https://doi.org/10.1016/j.cub.2007.02.027 
1091

1092

1093

1094

1095

1096

1097

1098

1099

1100

1101

1102

1103

1104

1105

1106

1107

1108

1109

1110

1111

1112

1113

1114

1115

1116

1117

1118

1119

1120

1121

1122

1123

1124

1125

1126

1127

1128

1129

1130

1131

1132

1133

1134

1135

1136

1137

1138

1139

1140

1141

Cinalli, R.M., Rangan, P., Lehmann, R., 2008. Germ cells are forever. Cell 132, 559-562.

Clough, E., Moon, W., Wang, S., Smith, K., Hazelrigg, T., 2007. Histone methylation is required for oogenesis in Drosophila. Development 134, 157-165. https://doi.org/10.1242/dev.02698

Clough, E., Tedeschi, T., Hazelrigg, T., 2014. Epigenetic regulation of oogenesis and germ stem cell maintenance by the Drosophila histone methyltransferase Eggless/dSetDB1. Developmental Biology 388, 181-191. https://doi.org/10.1016/j.ydbio.2014.01.014

Colozza, G., Montembault, E., Quénerch'du, E., Riparbelli, M.G., D’Avino, P.P., Callaini, G., 2011. Drosophila nucleoporin Nup154 controls cell viability, proliferation and nuclear accumulation of Mad transcription factor. Tissue and Cell 43, 254-261. https://doi.org/10.1016/j.tice.2011.05.001

Czech, B., Munafò, M., Ciabrelli, F., Eastwood, E.L., Fabry, M.H., Kneuss, E., Hannon, G.J., 2018. piRNA-Guided Genome Defense: From Biogenesis to Silencing. Annu Rev Genet 52, 131-157. https://doi.org/10.1146/annurev-genet-120417-031441

Dansereau, D.A., Lasko, P., 2008. The Development of Germline Stem Cells in Drosophila. Methods Mol Biol 450, 3-26. https://doi.org/10.1007/978-1-60327-214-8_1

Davis, L.I., Blobel, G., 1987. Nuclear pore complex contains a family of glycoproteins that includes p62: glycosylation through a previously unidentified cellular pathway. PNAS 84, 7552-7556. https://doi.org/10.1073/pnas.84.21.7552

Devlin, R.H., Bingham, B., Wakimoto, B.T., 1990. The organization and expression of the light gene, a heterochromatic gene of Drosophila melanogaster. Genetics 125, 129-140.

Doucet, C.M., Hetzer, M.W., 2010. Nuclear pore biogenesis into an intact nuclear envelope. Chromosoma 119, 469-477. https://doi.org/10.1007/s00412-010-0289-2

Duan, T., Green, N., Tootle, T.L., Geyer, P.K., 2020. Nuclear architecture as an intrinsic regulator of Drosophila female germline stem cell maintenance. Current Opinion in Insect Science, Development and regulation 37, 30-38. https://doi.org/10.1016/j.cois.2019.11.007

Eymery, A., Liu, Z., Ozonov, E.A., Stadler, M.B., Peters, A.H.F.M., 2016. The methyltransferase Setdb1 is essential for meiosis and mitosis in mouse oocytes and early embryos. Development 143, 2767-2779. https://doi.org/10.1242/dev.132746

Flora, P., McCarthy, A., Upadhyay, M., Rangan, P., 2017. Role of chromatin modifications in Drosophila germline stem cell differentiation, in: Results and Problems in Cell Differentiation. pp. 1-30. https://doi.org/10.1007/978-3-319-44820-6_1

Flora, P., Schowalter, S., Wong-Deyrup, S., DeGennaro, M., Nasrallah, M.A., Rangan, P., 2018. Transient transcriptional silencing alters the cell cycle to promote germline stem cell differentiation in Drosophila. Developmental biology 434, 84-95.

Frietze, S., O'Geen, H., Blahnik, K.R., Jin, V.X., Farnham, P.J., 2010. ZNF274 Recruits the Histone Methyltransferase SETDB1 to the 3' Ends of ZNF Genes. PLOS ONE 5, e15082. https://doi.org/10.1371/journal.pone.0015082

Gerbasi, V.R., Preall, J.B., Golden, D.E., Powell, D.W., Cummins, T.D., Sontheimer, E.J., 2011. Blanks, a nuclear siRNA/dsRNA-binding complex component, is required for Drosophila spermiogenesis. PNAS 108, 3204-3209. https://doi.org/10.1073/pnas.1009781108

Gigliotti, S., Callaini, G., Andone, S., Riparbelli, M.G., Pernas-Alonso, R., Hoffmann, G., Graziani, F., Malva, C., 1998. Nup154, a new Drosophila gene essential for male and female gametogenesis is related to the nup155 vertebrate nucleoporin gene. J Cell Biol 142, 1195-1207. https://doi.org/10.1083/jcb.142.5.1195

Gilboa, L., Lehmann, R., 2004. Repression of primordial germ cell differentiation parallels germ line stem cell maintenance. Curr Biol 14, 981-986. https://doi.org/10.1016/j.cub.2004.05.049

Gozalo, A., Capelson, M., 2016. A New Path through the Nuclear Pore. Cell 167, 1159-1160. https://doi.org/10.1016/j.cell.2016.11.011 
1142

1143

1144

1145

1146

1147

1148

1149

1150

1151

1152

1153

1154

1155

1156

1157

1158

1159

1160

1161

1162

1163

1164

1165

1166

1167

1168

1169

1170

1171

1172

1173

1174

1175

1176

1177

1178

1179

1180

1181

1182

1183

1184

1185

1186

1187

1188

1189

1190

1191

1192

Grimaldi, M.R., Cozzolino, L., Malva, C., Graziani, F., Gigliotti, S., 2007. nup154 genetically interacts with cup and plays a cell-type-specific function during Drosophila melanogaster egg-chamber development. Genetics 175, 1751-1759. https://doi.org/10.1534/genetics.106.062844

Güttinger, S., Laurell, E., Kutay, U., 2009. Orchestrating nuclear envelope disassembly and reassembly during mitosis. Nat Rev Mol Cell Biol 10, 178-191. https://doi.org/10.1038/nrm2641

Hampoelz, B., Schwarz, A., Ronchi, P., Bragulat-Teixidor, H., Tischer, C., Gaspar, I., Ephrussi, A., Schwab, Y., Beck, M., 2019. Nuclear Pores Assemble from Nucleoporin Condensates During Oogenesis. Cell 179, 671-686.e17. https://doi.org/10.1016/j.cell.2019.09.022

Holla, S., Dhakshnamoorthy, J., Folco, H.D., Balachandran, V., Xiao, H., Sun, L., Wheeler, D., Zofall, M., Grewal, S.I.S., 2020. Positioning heterochromatin at the nuclear periphery suppresses histone turnover to promote epigenetic inheritance. Cell 180, 150-164.e15. https://doi.org/10.1016/j.cell.2019.12.004

Hou, C., Corces, V.G., 2010. Nups take leave of the nuclear envelope to regulate transcription. Cell 140, 306-308. https://doi.org/10.1016/j.cell.2010.01.036

Hughes, S.E., Miller, D.E., Miller, A.L., Hawley, R.S., 2018. Female Meiosis: Synapsis, Recombination, and Segregation in Drosophila melanogaster. Genetics 208, 875-908. https://doi.org/10.1534/genetics.117.300081

Huynh, J.-R., St Johnston, D., 2004. The Origin of Asymmetry: Early Polarisation of the Drosophila Germline Cyst and Oocyte. Current Biology 14, R438-R449. https://doi.org/10.1016/j.cub.2004.05.040

Iglesias, N., Paulo, J.A., Tatarakis, A., Wang, X., Edwards, A.L., Bhanu, N.V., Garcia, B.A., Haas, W., Gygi, S.P., Moazed, D., 2020. Native Chromatin Proteomics Reveals Role for Specific Nucleoporins in Heterochromatin Organization and Maintenance. Mol Cell 77, 51-66.e8. https://doi.org/10.1016/j.molcel.2019.10.018

Jevitt, A., Chatterjee, D., Xie, G., Wang, X.-F., Otwell, T., Huang, Y.-C., Deng, W.-M., 2020. A single-cell atlas of adult Drosophila ovary identifies transcriptional programs and somatic cell lineage regulating oogenesis. PLOS Biology 18, e3000538. https://doi.org/10.1371/journal.pbio.3000538

Katsani, K.R., Karess, R.E., Dostatni, N., Doye, V., 2008. In Vivo Dynamics of Drosophila Nuclear Envelope Components. MBoC 19, 3652-3666. https://doi.org/10.1091/mbc.e07$11-1162$

Kershner, A., Crittenden, S.L., Friend, K., Sorensen, E.B., Porter, D.F., Kimble, J., 2013. Germline Stem Cells and Their Regulation in the Nematode Caenorhabditis elegans, in: Hime, G., Abud, H. (Eds.), Transcriptional and Translational Regulation of Stem Cells, Advances in Experimental Medicine and Biology. Springer Netherlands, Dordrecht, pp. 29-46. https://doi.org/10.1007/978-94-007-6621-1_3

Kiseleva, E., Rutherford, S., Cotter, L.M., Allen, T.D., Goldberg, M.W., 2001. Steps of nuclear pore complex disassembly and reassembly during mitosis in early Drosophila embryos. Journal of Cell Science 114, 3607-3618. https://doi.org/10.1242/jcs.114.20.3607

Ko, K., Araúzo-Bravo, M.J., Kim, J., Stehling, M., Schöler, H.R., 2010. Conversion of adult mouse unipotent germline stem cells into pluripotent stem cells. Nat Protoc 5, 921-928. https://doi.org/10.1038/nprot.2010.44

Koch, C.M., Honemann-Capito, M., Egger-Adam, D., Wodarz, A., 2009. Windei, the Drosophila Homolog of mAM/MCAF1, Is an Essential Cofactor of the H3K9 Methyl Transferase dSETDB1/Eggless in Germ Line Development. PLoS Genet 5, e1000644. https://doi.org/10.1371/journal.pgen.1000644

Koch, E.A., Smith, P.A., King, R.C., 1967. The division and differentiation of Drosophila cystocytes. J. Morphol. 121, 55-70. https://doi.org/10.1002/jmor.1051210106 
1193

1194

1195

1196

1197

1198

1199

1200

1201

1202

1203

1204

1205

1206

1207

1208

1209

1210

1211

1212

1213

1214

1215

1216

1217

1218

1219

1220

1221

1222

1223

1224

1225

1226

1227

1228

1229

1230

1231

1232

1233

1234

1235

1236

1237

1238

1239

1240

1241

1242

Kugler, J.-M., Lasko, P., 2009. Localization, anchoring and translational control of oskar, gurken, bicoid and nanos mRNA during Drosophila oogenesis. Fly (Austin) 3, 15-28. https://doi.org/10.4161/fly.3.1.7751

Kuhn, T.M., Pascual-Garcia, P., Gozalo, A., Little, S.C., Capelson, M., 2019. Chromatin targeting of nuclear pore proteins induces chromatin decondensation. Journal of Cell Biology 218, 2945-2961. https://doi.org/10.1083/jcb.201807139

Kutay, U., Jühlen, R., Antonin, W., 2021. Mitotic disassembly and reassembly of nuclear pore complexes. Trends in Cell Biology. https://doi.org/10.1016/j.tcb.2021.06.011

Lasko, P.F., Ashburner, M., 1988. The product of the Drosophila gene vasa is very similar to eukaryotic initiation factor-4A. Nature 335, 611-617. https://doi.org/10.1038/335611a0

Laurell, E., Kutay, U., 2011. Dismantling the NPC permeability barrier at the onset of mitosis. Cell Cycle 10, 2243-2245. https://doi.org/10.4161/cc.10.14.16195

Lehmann, R., 2012. Germline Stem Cells: Origin and Destiny. Cell Stem Cell 10, 729-739. https://doi.org/10.1016/j.stem.2012.05.016

Lesch, B.J., Page, D.C., 2012. Genetics of germ cell development. Nature Reviews Genetics. https://doi.org/10.1038/nrg3294

Lilly, M.A., Spradling, A.C., 1996. The Drosophila endocycle is controlled by Cyclin E and lacks a checkpoint ensuring S-phase completion. Genes Dev 10, 2514-2526. https://doi.org/10.1101/gad.10.19.2514

Mach, J.M., Lehmann, R., 1997. An Egalitarian-BicaudalD complex is essential for oocyte specification and axis determination in Drosophila. Genes Dev. 11, 423-435. https://doi.org/10.1101/gad.11.4.423

Malone, C.D., Brennecke, J., Dus, M., Stark, A., McCombie, W.R., Sachidanandam, R., Hannon, G.J., 2009. Specialized piRNA Pathways Act in Germline and Somatic Tissues of the Drosophila Ovary. Cell 137, 522-535. https://doi.org/10.1016/j.cell.2009.03.040

McCarthy, A., Sarkar, K., Martin, E.T., Upadhyay, M., James, J.R., Lin, J.M., Jang, S., Williams, N.D., Forni, P.E., Buszczak, M., Rangan, P., 2019. MSL3 coordinates a transcriptional and translational meiotic program in female Drosophila. bioRxiv 2019.12.18.879874. https://doi.org/10.1101/2019.12.18.879874

McCloskey, A., Ibarra, A., Hetzer, M.W., 2018. Tpr regulates the total number of nuclear pore complexes per cell nucleus. Genes Dev. 32, 1321-1331. https://doi.org/10.1101/gad.315523.118

McKearin, D., Ohlstein, B., 1995. A role for the Drosophila bag-of-marbles protein in the differentiation of cystoblasts from germline stem cells. Development (Cambridge, England) 121, 2937-2947.

McKearin, D.M., Spradling, A.C., 1990. bag-of-marbles: a Drosophila gene required to initiate both male and female gametogenesis. Genes \& development 4, 2242-2251.

Navarro, C., Puthalakath, H., Adams, J.M., Strasser, A., Lehmann, R., 2004. Egalitarian binds dynein light chain to establish oocyte polarity and maintain oocyte fate. Nat Cell Biol 6, 427-435. https://doi.org/10.1038/ncb1122

Orr-Weaver, T.L., 1995. Meiosis in Drosophila: seeing is believing. Proceedings of the National Academy of Sciences of the United States of America 92, 10443-10449.

Osumi, K., Sato, K., Murano, K., Siomi, H., Siomi, M.C., 2019. Essential roles of Windei and nuclear monoubiquitination of Eggless/SETDB1 in transposon silencing. EMBO Rep 20, e48296. https://doi.org/10.15252/embr.201948296

Page, S.L., Hawley, R.S., 2001. c(3)G encodes a Drosophila synaptonemal complex protein. Genes \& development 15, 3130-3143.

Rangan, P., Malone, C.D., Navarro, C., Newbold, S.P., Hayes, P.S., Sachidanandam, R., Hannon, G.J., Lehmann, R., 2011. piRNA production requires heterochromatin formation in Drosophila. Curr Biol 21, 1373-1379. https://doi.org/10.1016/j.cub.2011.06.057 
1243

1244

1245

1246

1247

1248

1249

1250

1251

1252

1253

1254

1255

1256

1257

1258

1259

1260

1261

1262

1263

1264

1265

1266

1267

1268

1269

1270

1271

1272

1273

1274

1275

1276

1277

1278

1279

1280

1281

1282

1283

1284

1285

1286

1287

1288

1289

1290

1291

1292

Reik, W., Surani, M.A., 2015. Germline and Pluripotent Stem Cells. Cold Spring Harb Perspect Biol 7, a019422. https://doi.org/10.1101/cshperspect.a019422

Riparbelli, M.G., Gottardo, M., Callaini, G., 2017. Parthenogenesis in Insects: The Centriole Renaissance. Results Probl Cell Differ 63, 435-479. https://doi.org/10.1007/978-3-31960855-6_19

Sarma, N.J., Willis, K., 2012. The new nucleoporin: regulator of transcriptional repression and beyond. Nucleus 3, 508-515. https://doi.org/10.4161/nucl.22427

Schultz, D.C., Ayyanathan, K., Negorev, D., Maul, G.G., Rauscher, F.J., 2002. SETDB1: a novel KAP-1-associated histone H3, lysine 9-specific methyltransferase that contributes to HP1-mediated silencing of euchromatic genes by KRAB zinc-finger proteins. Genes Dev 16, 919-932. https://doi.org/10.1101/gad.973302

Schwartz, Y.B., Cavalli, G., 2017. Three-Dimensional Genome Organization and Function in Drosophila. Genetics 205, 5-24. https://doi.org/10.1534/genetics.115.185132

Seum, C., Reo, E., Peng, H., Rauscher, F.J., Spierer, P., Bontron, S., 2007. Drosophila SETDB1 Is Required for Chromosome 4 Silencing. PLoS Genet 3, e76. https://doi.org/10.1371/journal.pgen.0030076

Seydoux, G., Braun, R.E., 2006. Pathway to totipotency: lessons from germ cells. Cell 127, 891-904. https://doi.org/10.1016/j.cell.2006.11.016

Shapiro-Kulnane, L., Smolko, A.E., Salz, H.K., 2015. Maintenance of Drosophila germline stem cell sexual identity in oogenesis and tumorigenesis. Development 142, 1073-1082. https://doi.org/10.1242/dev.116590

Sienski, G., Dönertas, D., Brennecke, J., 2012. Transcriptional Silencing of Transposons by Piwi and Maelstrom and Its Impact on Chromatin State and Gene Expression. Cell 151, 964980. https://doi.org/10.1016/j.cell.2012.10.040

Skene, P.J., Henikoff, S., 2017. An efficient targeted nuclease strategy for high-resolution mapping of DNA binding sites. eLife 6, e21856. https://doi.org/10.7554/eLife.21856

Smolko, A.E., Shapiro-Kulnane, L., Salz, H.K., 2020. An autoregulatory switch in sex-specific phf7 transcription causes loss of sexual identity and tumors in the Drosophila female germline. Development 147. https://doi.org/10.1242/dev.192856

Smolko, A.E., Shapiro-Kulnane, L., Salz, H.K., 2018. The H3K9 methyltransferase SETDB1 maintains female identity in Drosophila germ cells. Nat Commun 9, 4155. https://doi.org/10.1038/s41467-018-06697-x

Sood, V., Brickner, J.H., 2014. Nuclear pore interactions with the genome. Curr Opin Genet Dev 25, 43-49. https://doi.org/10.1016/j.gde.2013.11.018

Spradling, A., Fuller, M.T., Braun, R.E., Yoshida, S., 2011. Germline Stem Cells. Cold Spring Harb Perspect Biol 3, a002642. https://doi.org/10.1101/cshperspect.a002642

Spradling, Allan C, 1993. Developmental genetics of oogenesis, in: The Development of Drosophila Melanogaster.

Spradling, A C, 1993. Germline cysts: communes that work. Cell 72, 649-651.

Telfer, W.H., 1975. Development and Physiology of the Oöcyte-Nurse Cell Syncytium. Advances in Insect Physiology. https://doi.org/10.1016/S0065-2806(08)60164-2

Timms, R.T., Tchasovnikarova, I.A., Antrobus, R., Dougan, G., Lehner, P.J., 2016. ATF7IPMediated Stabilization of the Histone Methyltransferase SETDB1 Is Essential for Heterochromatin Formation by the HUSH Complex. Cell Rep 17, 653-659. https://doi.org/10.1016/j.celrep.2016.09.050

Tsusaka, T., Shimura, C., Shinkai, Y., 2019. ATF7IP regulates SETDB1 nuclear localization and increases its ubiquitination. EMBO Rep 20, e48297. https://doi.org/10.15252/embr.201948297

Upadhyay, M., Martino Cortez, Y., Wong-Deyrup, S., Tavares, L., Schowalter, S., Flora, P., Hill, C., Nasrallah, M.A., Chittur, S., Rangan, P., 2016. Transposon Dysregulation Modulates 
dWnt4 Signaling to Control Germline Stem Cell Differentiation in Drosophila. PLoS genetics 12, e1005918.

Valm, A.M., Cohen, S., Legant, W.R., Melunis, J., Hershberg, U., Wait, E., Cohen, A.R., Davidson, M.W., Betzig, E., Lippincott-Schwartz, J., 2017. Applying systems-level spectral imaging and analysis to reveal the organelle interactome. Nature 546, 162-167. https://doi.org/10.1038/nature22369

Wang, W., Han, B.W., Tipping, C., Ge, D.T., Zhang, Z., Weng, Z., Zamore, P.D., 2015. Slicing and Binding by Ago 3 or Aub Trigger Piwi-Bound piRNA Production by Distinct Mechanisms. Mol Cell 59, 819-830. https://doi.org/10.1016/j.molcel.2015.08.007

Weiler, K.S., Wakimoto, B.T., 1995. Heterochromatin and gene expression in Drosophila. Annu Rev Genet 29, 577-605. https://doi.org/10.1146/annurev.ge.29.120195.003045

Wilson, J.E., Connell, J.E., Macdonald, P.M., 1996. aubergine enhances oskar translation in the Drosophila ovary. Development 122, 1631-1639. https://doi.org/10.1242/dev.122.5.1631

Xie, T., 2013. Control of germline stem cell self-renewal and differentiation in the Drosophila ovary: concerted actions of niche signals and intrinsic factors. Wiley Interdiscip Rev Dev Biol 2, 261-273. https://doi.org/10.1002/wdev.60

Xie, T., Spradling, A.C., 2000. A niche maintaining germ line stem cells in the Drosophila ovary. Science 290, 328-330. https://doi.org/10.1126/science.290.5490.328

Yoon, J., Lee, K.-S., Park, J.S., Yu, K., Paik, S.-G., Kang, Y.-K., 2008. dSETDB1 and SU(VAR)3-9 Sequentially Function during Germline-Stem Cell Differentiation in Drosophila melanogaster. PLOS ONE 3, e2234. https://doi.org/10.1371/journal.pone.0002234

Yuan, H., Yamashita, Y.M., 2010. Germline stem cells: stems of the next generation. Curr Opin Cell Biol 22, 730-736. https://doi.org/10.1016/j.ceb.2010.08.013

Zaccai, M., Lipshitz, H.D., 1996. Differential distributions of two adducin-like protein isoforms in the Drosophila ovary and early embryo. Zygote 4, 159-166. https://doi.org/10.1017/s096719940000304x 